\title{
Observational constraints on sign-changeable interaction models and alleviation of the $H_{0}$ tension
}

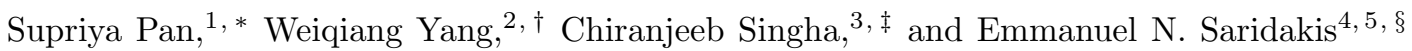 \\ ${ }^{1}$ Department of Mathematics, Presidency University, 86/1 College Street, Kolkata 700073, India \\ ${ }^{2}$ Department of Physics, Liaoning Normal University, Dalian, 116029, P. R. China \\ ${ }^{3}$ Department of Physical Sciences, Indian Institute of Science Education \\ and Research Kolkata, Mohanpur 741246, West Bengal, India \\ ${ }^{4}$ Department of Physics, National Technical University of Athens, Zografou Campus GR 157 73, Athens, Greece \\ ${ }^{5}$ Department of Astronomy, School of Physical Sciences, \\ University of Science and Technology of China, Hefei 230026, P.R. China
}

\begin{abstract}
We investigate various scenarios which include interaction forms between dark matter and dark energy that exhibit sign reverse, namely where the transfer of energy between the dark fluids changes sign during evolution. We study the large-scale inhomogeneities in such interacting scenarios and we confront them with the latest astronomical data. Our analysis shows that the sign-changeable interaction models are able to produce stable perturbations. Additionally, the data seem to slightly favor a non-zero interaction, however, within $1 \sigma$ confidence level (CL) the scenarios cannot be distinguished from non-interacting cosmologies. We find that the best-fit value of the dark-energy equation-of-state parameter lies in the phantom regime, while the quintessence region is also allowed nevertheless at more than $2 \sigma$ CL. Examining the effect of the interaction on the CMB TT and matter power spectra we show that while from the simple spectra it is hard to distinguish the interacting case from $\Lambda \mathrm{CDM}$ scenario, in the residual graphs the interaction is indeed traceable. Moreover, we find that sign-changeable interaction models can reconcile the $H_{0}$ tension, however the $\sigma_{8}$ tension is still persisting. Finally, we examine the validity of the laws of thermodynamics and we show that the generalized second law is always satisfied, while the second derivative of the total entropy becomes negative at late times which implies that the universe tends towards thermodynamic equilibrium.
\end{abstract}

PACS numbers: 98.80.-k, 95.36.+x, 95.35.+d, 98.80.Es

\section{INTRODUCTION}

Recent observations of various origin suggest that around $69 \%$ of the universe consists of the dark energy sector, while around $26 \%$ constitutes of the cold dark matter one [1. One usually assumes that these two sectors do not mutually interact, and the resulting scenario is capable of describing very efficiently the various sets of independent observations. Nevertheless, since the underlying microscopic theory of both these sectors is unknown, there is not any field-theoretical argument against the consideration of a possible mutual interaction. Furthermore, such an interaction could alleviate the known coincidence problem [2, 3, 4, namely why are the energy densities of dark matter and dark energy currently of the same order although they follow completely different scaling laws during evolution. Hence, in the literature one can find many such interacting scenarios (see [5, 6] for review and references therein), independently of the specific dark-energy nature, namely whether it arises from fields [7, 8, or through a gravitational modification [9, 10, 11. The interacting scenarios can be very efficient in describing late-time universe, and moreover they seem

\footnotetext{
*Electronic address: supriya.maths@presiuniv.ac.in

${ }^{\dagger}$ Electronic address: d11102004@163.com

‡Electronic address: cs12ip026@iiserkol.ac.in

$\S$ Electronic address: msaridak@phys.uoa.gr
}

to be slightly favored comparing to non-interacting ones [12, 13, 14, 15, 16, 17, 18, 19, 20, 21, 22, 23, 24, 25, 26. 27, 28, 29. Additionally, they seem to be efficient in addressing the $H_{0}$ tension [30, 31, 32, 33, 34] as well as the $\sigma_{8}$ tension [34, 35, 36].

In the above scenarios the interaction term, that determines the interaction rate and thus the flow of energy between the dark matter and dark energy sectors, is introduced phenomenologically. Although there is a large variety in such choices, they are usually assumed to have the same sign, namely the energy flow is from one component to the other during the whole universe evolution. However, an interesting question arises, namely what would happen if we allow for a sign change of the interaction term during the cosmological evolution. Such a consideration might be further useful to investigate the dark sectors' physics. Hence, in the present work we desire to investigate this possibility and in particular to confront the obtained scenarios with different observational data coming from probes like the cosmic microwave background radiation (CMB), supernovae type Ia (SNIa), baryon acoustic oscillations (BAO), and Hubble parameter measurements.

We organize the present work in the following way. In Section [I] we provide the cosmological equations at background and perturbative levels, in presence of arbitrary coupling in the dark sector. In Section III we present the models that we wish to study in this work. In Section IV we describe the observational datasets and the fitting 
methodology. In Section $\mathrm{V}$ we provide the constraints on the models, and we perform a Bayesian analysis in comparison with $\Lambda \mathrm{CDM}$ cosmology. Finally, we close the present work in Section VII with a brief summary of the results.

\section{INTERACTING COSMOLOGY}

In this Section we briefly review interacting cosmology. We consider a homogeneous and isotropic flat FriedmannRobertson-Walker (FRW) line element of the form

$$
d s^{2}=-d t^{2}+a^{2}(t) \delta_{i j} d x^{i} d x^{j},
$$

where $a(t)$ is the expansion scale factor of the universe. Furthermore, we consider the universe to be filled with baryons, cold dark matter, radiation, and the dark energy sector (which may be of effective origin or not), all of which considered as barotropic perfect fluids. Thus, the Friedmann equations that determine the universe evolution are written as

$$
\begin{aligned}
& H^{2}=\frac{8 \pi G}{3} \rho_{t}, \\
& 2 \dot{H}+3 H^{2}=-8 \pi G p_{t},
\end{aligned}
$$

with $G$ the Newton's constant and $H=\dot{a} / a$ the Hubble function (dots denote derivatives with respect to $t$ ). In the above equations we have introduced the total energy density and pressure respectively as $\rho_{t}=\rho_{r}+\rho_{b}+\rho_{c}+\rho_{x}$ and $p_{t}=p_{r}+p_{b}+p_{c}+p_{x}$, with the subscripts $r, b, c, x$ denoting radiation, baryon, cold dark matter and dark energy.

Although the Bianchi identities lead to the conservations of the total energy momentum tensor, they do not imply anything for the separate sectors, and thus one can assume that some of them mutually interact [2, 3, 4, 37. In this work we allow the dark matter and dark energy sectors to interact, while radiations and baryonic matter are considered to be conserved. In particular, we assume that

$$
\begin{aligned}
& \dot{\rho}_{b}+3 H \rho_{b}=0 \\
& \dot{\rho}_{r}+4 H \rho_{r}=0 \\
& \dot{\rho}_{c}+3 H \rho_{c}=-Q, \\
& \dot{\rho}_{x}+3 H\left(1+w_{x}\right) \rho_{x}=Q,
\end{aligned}
$$

where $w_{x}$ is the dark-energy equation-of-state parameter (for baryonic and dark matter we consider the dust case $w_{b}=w_{c}=0$, while for radiation as usual $w_{r}=1 / 3$ ). The introduced quantity $Q$ is a phenomenological descriptor of the interaction, and its form is considered arbitrarily. If $Q>0$ then the energy transfer is from cold dark matter (pressureless dark matter) to dark energy, while if $Q<0$ then it is from dark energy to dark matter. Moreover, as usual the conservation equations for baryonic matter and radiation give $\rho_{b}=\rho_{b 0} a^{-3}$ and $\rho_{r}=\rho_{r 0} a^{-4}$ respectively, with $\rho_{i 0}(i=r, b)$ the value of $\rho_{i}$ at present time. In summary, if the interaction function is given then the Friedmann equation (2) alongside the conservation equations (6) and (7), can determine the evolution of the universe.

One can see that the conservation equations (6) and 77) can be written in an alternative way as

$$
\begin{aligned}
& \dot{\rho}_{c}+3 H\left(1+w_{c}^{\text {eff }}\right) \rho_{c}=0, \\
& \dot{\rho}_{x}+3 H\left(1+w_{x}^{\text {eff }}\right) \rho_{x}=0,
\end{aligned}
$$

where $w_{c}^{\text {eff }}$, and $w_{x}^{\text {eff }}$ are the effective equation-of-state parameters for cold dark matter and dark energy, given as

$$
\begin{aligned}
& w_{c}^{\text {eff }}=\frac{Q}{3 H \rho_{c}}, \\
& w_{x}^{\text {eff }}=w_{x}-\frac{Q}{3 H \rho_{x}} .
\end{aligned}
$$

Hence, as we observe, the interaction affects the equation of state of these components. In particular, dark matter may depart from dust while dark energy may be quintessence or phantom like even if the initial $w_{x}$ is fixed to one regime.

We proceed to the investigation of the above scenarios at the level of perturbations. We consider scalar perturbations around an FRW metric given by [38, 39, 40.

$$
\begin{aligned}
d s^{2}=a^{2}(\tau)\{ & -(1+2 \phi) d \tau^{2}+2 \partial_{i} B d \tau d x^{i} \\
& \left.+\left[(1-2 \psi) \delta_{i j}+2 \partial_{i} \partial_{j} E\right] d x^{i} d x^{j}\right\},
\end{aligned}
$$

where $\tau$ represents the conformal time and the quantities $\phi, B, \psi, E$, denote the gauge-dependent scalar perturbations. Thus, in the case of interacting cosmology with $w_{x} \neq-1$ the perturbation equations in the synchronous gauge $\left(\phi=B=0, \psi=\eta\right.$, and $\left.k^{2} E=-h / 2-3 \eta\right)$, with $k$ the Fourier mode and $h, \eta$, being the metric scalar perturbations [39], are written as [41, 42, 43]:

$$
\begin{aligned}
\delta_{x}^{\prime}= & -\left(1+w_{x}\right)\left(\theta_{x}+\frac{h^{\prime}}{2}\right)-3 \mathcal{H} w_{x}^{\prime} \frac{\theta_{x}}{k^{2}} \\
& -3 \mathcal{H}\left(c_{s x}^{2}-w_{x}\right)\left[\delta_{x}+3 \mathcal{H}\left(1+w_{x}\right) \frac{\theta_{x}}{k^{2}}\right] \\
& +\frac{a Q}{\rho_{x}}\left[-\delta_{x}+\frac{\delta Q}{Q}+3 \mathcal{H}\left(c_{s x}^{2}-w_{x}\right) \frac{\theta_{x}}{k^{2}}\right], \\
\theta_{x}^{\prime}= & -\mathcal{H}\left(1-3 c_{s x}^{2}\right) \theta_{x}+\frac{c_{s x}^{2}}{\left(1+w_{x}\right)} k^{2} \delta_{x} \\
& +\frac{a Q}{\rho_{x}}\left[\frac{\theta_{c}-\left(1+c_{s x}^{2}\right) \theta_{x}}{1+w_{x}}\right], \\
\delta_{c}^{\prime}= & -\left(\theta_{c}+\frac{h^{\prime}}{2}\right)+\frac{a Q}{\rho_{c}}\left(\delta_{c}-\frac{\delta Q}{Q}\right), \\
\theta_{c}^{\prime}= & -\mathcal{H} \theta_{c} .
\end{aligned}
$$

In the above expressions we have introduced the overdensities $\delta_{i}=\delta \rho_{i} / \rho_{i}$, as well as the velocity perturbations $\theta_{i}$, with primes denoting derivatives with respect to the 
conformal time $\tau$, with $\mathcal{H}=\frac{a^{\prime}}{a}$ the conformal Hubble function. Moreover, $c_{s x}^{2}$ is the adiabatic sound speed, which in the following will be set to 1 [41, 42, 43] (the adiabatic sound speed for the dark matter in the dust case is $c_{s c}^{2}=0$ ).

Now, for the case where $w_{x}=-1$, namely in the case of interacting vacuum scenario, the perturbation equations are slightly different. In the synchronous gauge the momentum conservation equation for dark matter reduces to $\dot{\theta}_{c}=0$ [44, while the density perturbations can be recast into 44 .

$$
\dot{\delta}_{c}=-\frac{\dot{h}}{2}+\frac{Q}{\rho_{c}} \delta_{c}
$$

However, in this gauge the vacuum energy is spatially homogeneous, i.e. $\delta \rho_{x}=0$.

Finally, it is important to remark that although in the present work, as well as in many works in the literature, the spatial curvature of the universe is not considered, a series of works shows that the observational data do not exclude the non-zero curvature 45, 46, 47, 48, 49, 50, 51, 52, 53, and hence the general universe picture should include the spatial curvature as a free parameter. We will address such a complete investigation in a separate work.

\section{SIGN-CHANGEABLE INTERACTION MODELS}

Since the appearance of interacting cosmology, a variety of phenomenological coupling functions $Q$ have been introduced and investigated in the literature. In general, most of the coupling functions indicate a particular direction of energy transfer, namely either the transfer of energy from dark matter to dark energy or vice versa. This includes functions of the form $Q \propto H \rho_{c}$, $Q \propto H \rho_{x}, Q \propto H\left(\rho_{c}+\rho_{x}\right), Q \propto H\left(\rho_{c} \rho_{x}\right) /\left(\rho_{c}+\rho_{x}\right)$, $Q \propto H \rho_{x}^{2} /\left(\rho_{c}+\rho_{x}\right)$, nonlinear forms, etc [25, 32, 54, 55, 56, 57, 58, 59, 60, 61, 62, 63, 64, 65, 66, 67, 68, 69, 70, 71, 72, 73, 74, 75, 76, 77, 78, 79, 80, 81, 82, 83, 84, 85.

However, in principle one could also have the case in which the interaction function, i.e. $Q$, changes sign during the evolution, namely the energy transfer between the interacting sectors changes direction [86, 87, 88, 89, 90]. In the following subsections we examine two of such models separately.

\section{A. Interaction function $Q=3 H \xi\left(\rho_{c}-\rho_{x}\right)$}

The first interacting model in which the interacting function changes sign during evolution is

$$
Q(t)=3 H \xi\left(\rho_{c}-\rho_{x}\right)
$$

where $\xi$ is the coupling parameter. Note the interesting feature that when $\rho_{c}=\rho_{x}$ then the interaction becomes zero even if $\xi \neq 0$. This kind of feature was observed in a different interaction model [106]. Here, we consider
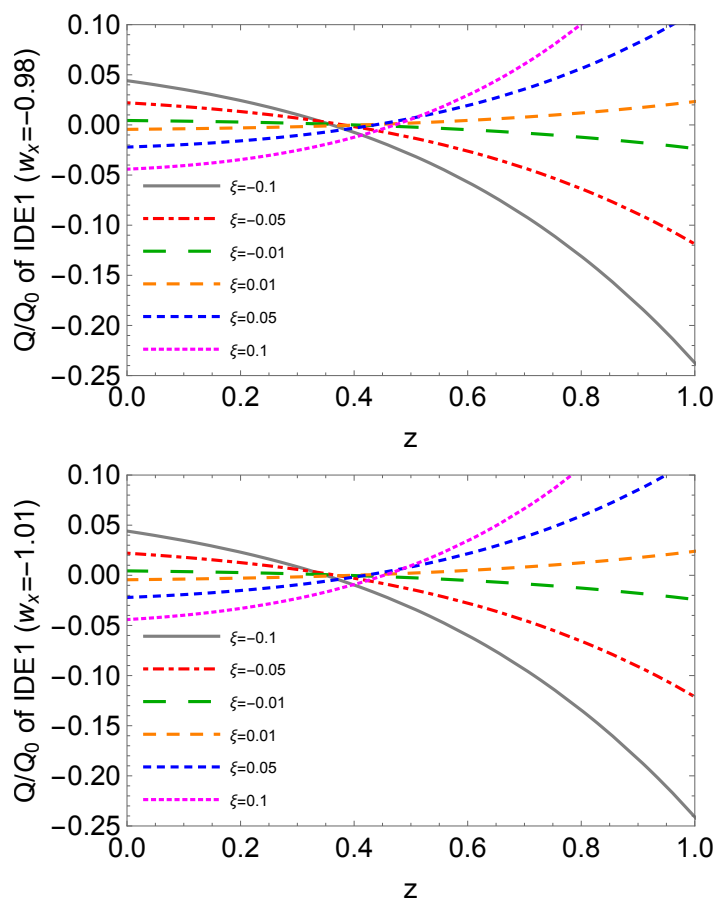

FIG. 1: The evolution of the normalized interaction function $Q / Q_{0}$, where $Q_{0}=H_{0} \rho_{t 0}$ (with $H_{0}$ and $\rho_{t 0}$ the present values of the Hubble parameter and the total energy density $\rho_{t}$ respectively), for the interaction model IDE1 of (18), for various values of the coupling parameter $\xi$ in units where $8 \pi G=1$, for $w_{x}=-0.98$ (upper graph) and for $w_{x}=-1.01$ (lower graph).

two choices for the dark energy equation-of-state parameter $w_{x}$, namely the cosmological constant case $w_{x}=-1$ (which corresponds to the interacting vacuum scenario, from now on model IVS1), and the case $w_{x} \neq-1$ (from now on model IDE1). The latter case is interesting since it allows to extract constraints on the dark energy equation-of-state parameter and examine whether it lies in the quintessence or in the phantom regime.

We proceed by numerically elaborating the background cosmological equations, using as independent variable the redshift, $z=a_{0} / a-1$, setting the present scale factor $a_{0}$ to 1 . Moreover, we introduce the density parameters of the various components through $\Omega_{i}=8 \pi G \rho_{i} /\left(3 H^{2}\right)$ and we set their current values as $\Omega_{x 0} \approx 0.69, \Omega_{c 0} \approx$ $0.25, \Omega_{b 0} \approx 0.05$ and $\Omega_{r 0} \approx 10^{-4}$ in agreement with observations [1].

In order to obtain a picture for the above interaction form, in Fig. 1 we first depict the evolution of $Q$ normalized by $Q_{0}=H_{0} \rho_{t 0}$ (with $H_{0}$ and $\rho_{t 0}$ the present values of the Hubble parameter and the total energy density $\rho_{t}$ respectively), for various values of $\xi$. As we can see, the sign-change direction and its exact moment depends on $\xi$, while the qualitative features of the interaction function do not change for $w_{x}>-1$ or $w_{x}<-1$. 

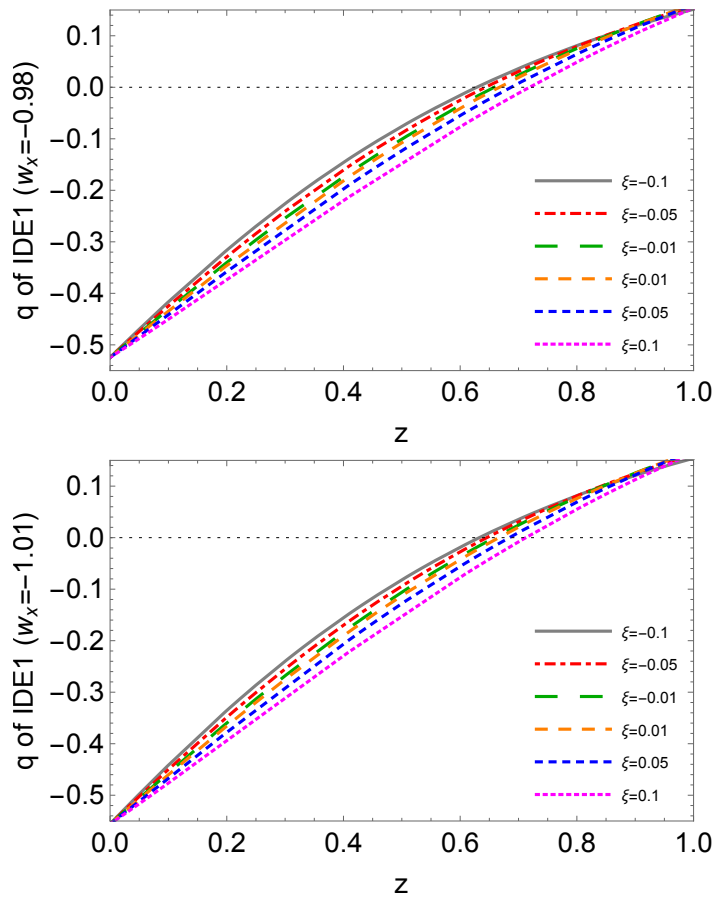

FIG. 2: The evolution of the deceleration parameter as a function of the redshift, for the interaction model IDE1 of (18), for $w_{x}=-0.98$ (upper graph) and for $w_{x}=-1.01$ (lower graph), for various values of the coupling parameter $\xi$ in units where $8 \pi G=1$. We have set $\Omega_{x 0} \approx 0.69, \Omega_{c 0} \approx 0.25, \Omega_{b 0} \approx 0.05$ and $\Omega_{r 0} \approx 10^{-4}$ in agreement with observations.

In Fig. 2 we depict the evolution of the deceleration parameter for model IDE1, for $w_{x}>-1$ and $w_{x}<-1$, and choosing various values of the coupling parameter $\xi$. As we observe, the value of $\xi$ may make faster or delay the transition from deceleration to acceleration, which is a significant advantage in fitting observations precisely. Additionally, note that the qualitative features do not change for either $w_{x}>-1$ or $w_{x}<-1$. A similar graph can be obtained in the case of model IVS1, namely when $w_{x}=-1$.

We mention that in this scenario one can extract the solution for the ratio $\frac{\rho_{x}}{\rho_{c}}$ analytically. In particular, inserting (18) into (6) and (7) gives

$$
\frac{\rho_{x}}{\rho_{c}}=-\frac{w_{x}}{2 \xi}-\sqrt{1+\frac{w_{x}^{2}}{4 \xi^{2}}}\left(\frac{1-b a^{3 \sqrt{4 \xi^{2}+w_{x}^{2}}}}{1+b a^{3 \sqrt{4 \xi^{2}+w_{x}^{2}}}}\right),
$$

where

$$
b \equiv-\frac{2 \xi \frac{\Omega_{x 0}}{\Omega_{c 0}}+w_{x}+\sqrt{4 \xi^{2}+w_{x}^{2}}}{2 \xi \frac{\Omega_{x 0}}{\Omega_{c 0}}+w_{x}-\sqrt{4 \xi^{2}+w_{x}^{2}}} .
$$

We proceed by investigating the coincidence parameter $r=\rho_{c} / \rho_{x}$ for this interaction model. As it is known, alleviation of the coincidence problem requires a non-zero value for $r$ at late times. The evolution of the coincidence parameter for the model IDE1 is presented in Fig.
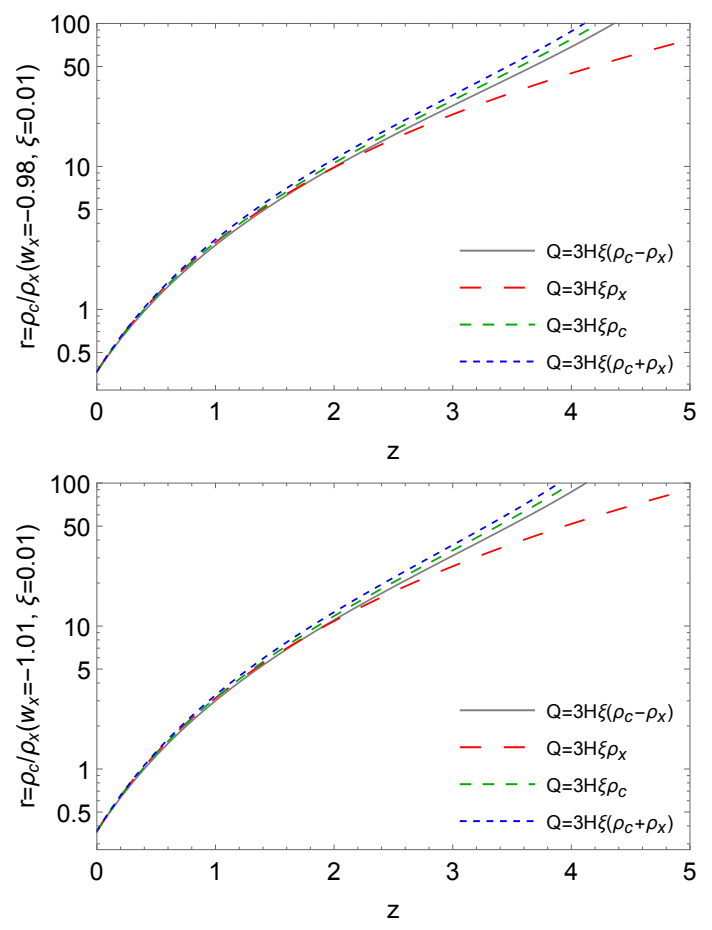

FIG. 3: The evolution of the coincidence parameter $r=$ $\rho_{c} / \rho_{x}$, for the interaction model IDE1 of $(18)$, for $w_{x}=-0.98$ (upper graph) and for $w_{x}=-1.01$ (lower graph), setting a typical value of $\xi=0.01$ in units where $8 \pi G=1$. For comparison we additionally depict the results for some well known interaction scenarios, namely $Q=3 H \xi \rho_{x}, Q=3 H \xi \rho_{c}$ and $Q=3 H \xi\left(\rho_{c}+\rho_{x}\right)$.

3. for different dark energy equation of states and setting a typical value of $\xi=0.01$ (for different values of $\xi$ the qualitative nature of the curves remains unaffected). Moreover, for comparison we also depict the results for some well known interaction scenarios of the literature, namely $Q=3 H \xi \rho_{x}, Q=3 H \xi \rho_{c}$ and $Q=3 H \xi\left(\rho_{c}+\rho_{x}\right)$. As one can see, as $z \rightarrow 0$ all curves tend to a non-zero value, which implies that the coincidence problem is alleviated. Additionally, although at low redshifts all models present the same behavior, at high redshifts the signchangeable interaction models considered in this work are distinguishable from the known interacting models of the literature.

Finally, concerning the perturbations in this scenario, they are determined by (13)-(16), with

$$
\frac{\delta Q}{Q}=\frac{\delta_{c} \rho_{c}-\delta_{x} \rho_{x}}{\rho_{c}-\rho_{x}}+\frac{2 \theta+h^{\prime}}{6 \mathcal{H}}
$$

with $\theta \equiv \theta_{\mu}^{\mu}$ the volume expansion of the total fluid. 

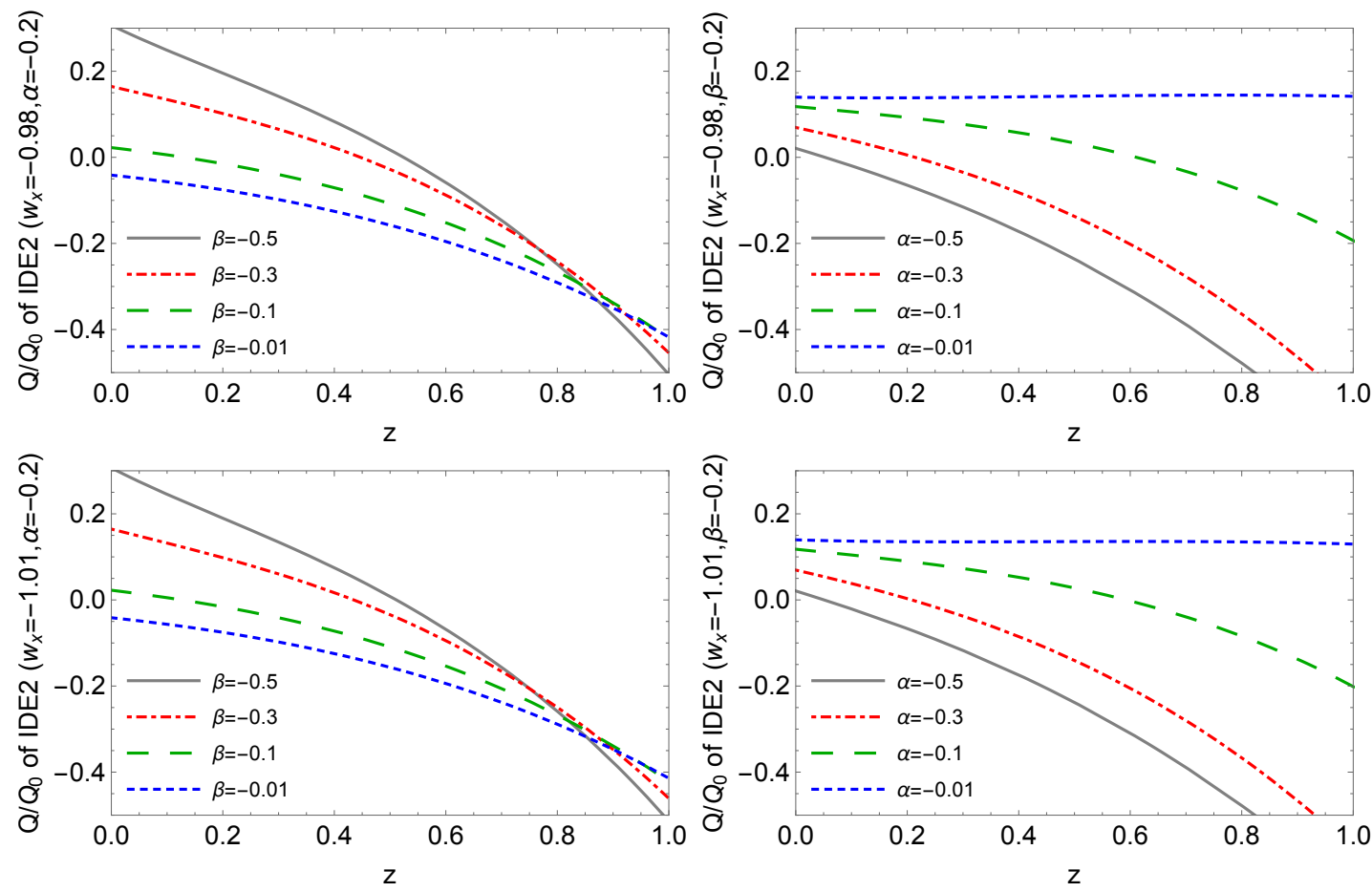

FIG. 4: The evolution of the normalized interaction function $Q / Q_{0}$, where $Q_{0}=H_{0} \rho_{t 0}$ (with $H_{0}$ and $\rho_{t 0}$ the present values of the Hubble parameter and the total energy density $\rho_{t}$ respectively), for the interaction model IDE2 of (22), for various values of the coupling parameters $\alpha$ and $\beta$ in units where $8 \pi G=1$, for $w_{x}=-0.98$ (upper graphs) and for $w_{x}=-1.01$ (lower graphs).
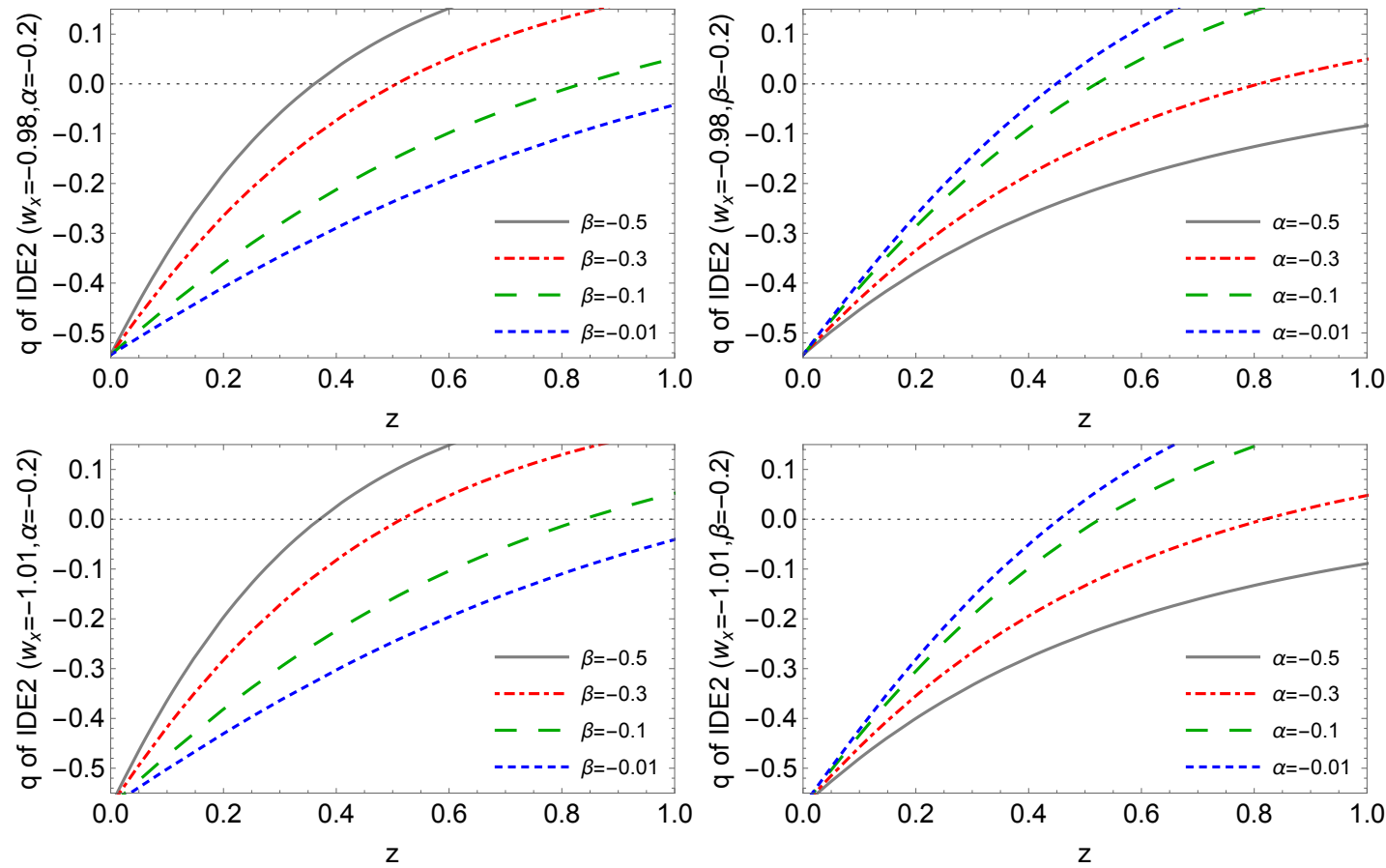

FIG. 5: The evolution of the deceleration parameter as a function of the redshift, for the interaction model IDE2 of (22), for $w_{x}=-0.98$ (upper graphs) and for $w_{x}=-1.01$ (lower graphs), for various values of the coupling parameters $\alpha$ and $\beta$ in units where $8 \pi G=1$. We have set $\Omega_{x 0} \approx 0.69, \Omega_{c 0} \approx 0.25, \Omega_{b 0} \approx 0.05$ and $\Omega_{r 0} \approx 10^{-4}$ in agreement with observations. 

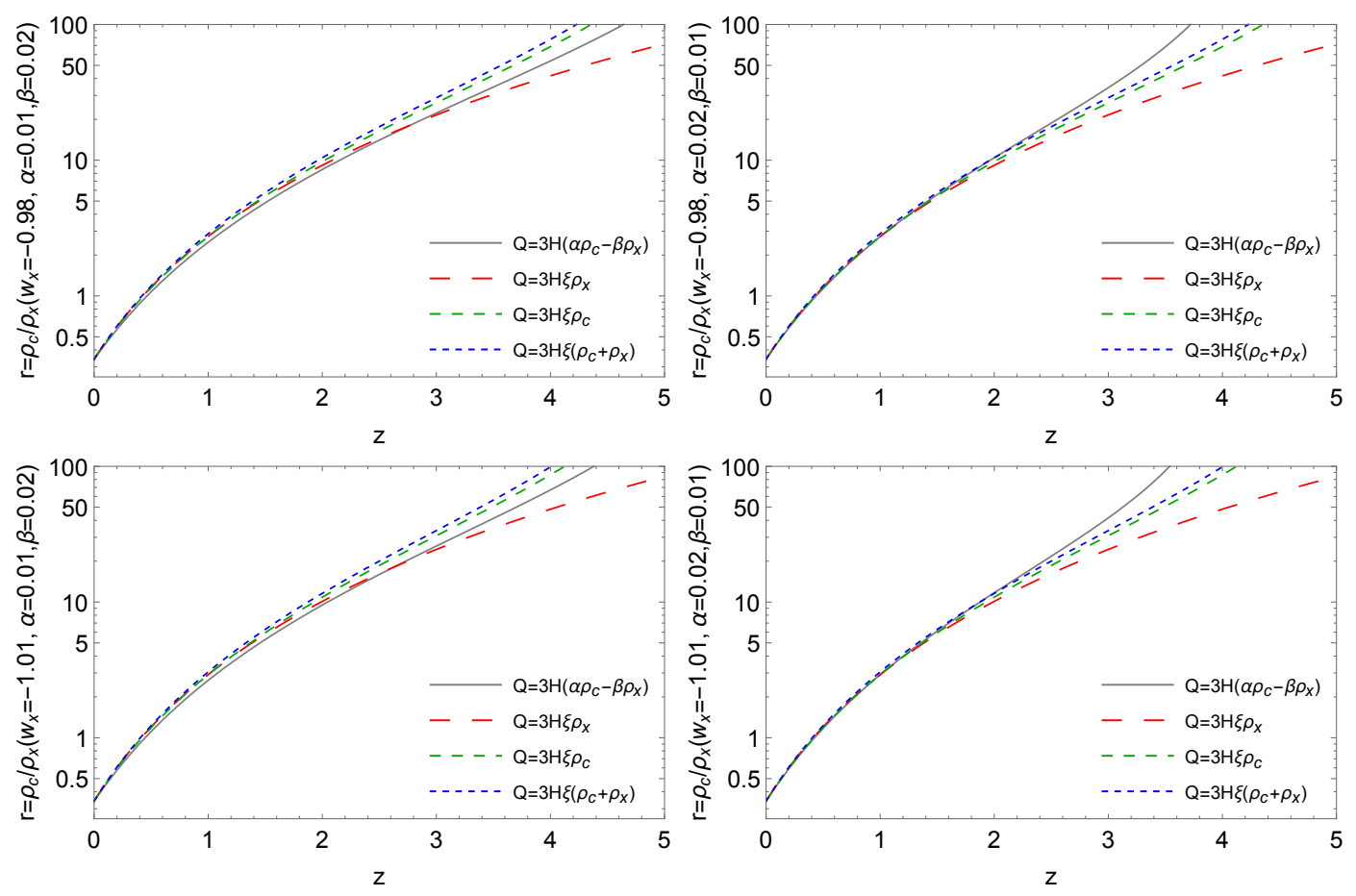

FIG. 6: The evolution of the coincidence parameter $r=\rho_{c} / \rho_{x}$, for the interaction model IDE2 of (22), for various values of the coupling parameters $\alpha$ and $\beta$ in units where $8 \pi G=1$, for $w_{x}=-0.98$ (upper graphs) and for $w_{x}=-1.01$ (lower graphs). For comparison we additionally depict the results for some well known interaction scenarios, namely $Q=3 H \xi \rho_{x}, Q=3 H \xi \rho_{c}$ and $Q=3 H \xi\left(\rho_{c}+\rho_{x}\right)$, taking a typical value $\xi=0.01$ in units where $8 \pi G=1$.

\section{B. Interaction function $Q=3 H\left(\alpha \rho_{c}-\beta \rho_{x}\right)$}

We proceed with the investigation of the interacting model

$$
Q=3 H\left(\alpha \rho_{c}-\beta \rho_{x}\right),
$$

where $\alpha, \beta$ are the coupling parameters considered to have the same sign. In the case where $\alpha=\beta=\xi$ the above model coincides with the one of the previous subsection, namely (18). We consider two choices for $w_{x}$, namely $w_{x}=-1$ (from now on model IVS2), and the case $w_{x} \neq-1$ (from now on model IDE2), which will be further divided into $w_{x}>-1$ and $w_{x}<-1$.

In Fig. 4 we depict the evolution of $Q$ normalized by $Q_{0}=H_{0} \rho_{t 0}$, for various values of the coupling parameters $\alpha$ and $\beta$. Additionally, in Fig. 5 we present the evolution of the deceleration parameter for model IDE2, for $w_{x}>-1$ and $w_{x}<-1$ and choosing various values of $\alpha$ and $\beta$. As we observe, the values of the coupling parameters significantly determine the exact transition redshift from deceleration to acceleration, while the exact value of $w_{x}$ does not have a significant effect. Similar graphs can be obtained in the case of IVS2 model, namely when $w_{x}=-1$.

Inserting $(22)$ into (6) and (7) provides as the analyt- ical solution for the ratio $\frac{\rho_{x}}{\rho_{c}}$, namely

$$
\begin{aligned}
\frac{\rho_{x}}{\rho_{c}}=\frac{\alpha-\beta-w_{x}}{2 \beta}- & \frac{\sqrt{\left(\alpha-\beta-w_{x}\right)^{2}+4 \alpha \beta}}{2 \beta} \\
& \cdot\left(\frac{1-b a^{3} \sqrt{\left(\alpha-\beta-w_{x}\right)^{2}+4 \alpha \beta}}{1+b a^{3 \sqrt{\left(\alpha-\beta-w_{x}\right)^{2}+4 \alpha \beta}}}\right),
\end{aligned}
$$

with

$$
b \equiv-\frac{2 \beta \frac{\Omega_{x 0}}{\Omega_{c 0}}-\left(\alpha-\beta-w_{x}\right)+\sqrt{\left(\alpha-\beta-w_{x}\right)^{2}+4 \alpha \beta}}{2 \beta \frac{\Omega_{x 0}}{\Omega_{c 0}}-\left(\alpha-\beta-w_{x}\right)-\sqrt{\left(\alpha-\beta-w_{x}\right)^{2}+4 \alpha \beta}} .
$$

We now investigate the evolution of the coincidence parameter $r=\rho_{c} / \rho_{x}$ for this interaction model. In Fig. 6. we depict the evolution of $r$ for various values of the coupling parameters $\alpha$ and $\beta$ and for different dark energy equation of states. Furthermore, for comparison we also depict the results for some well known interaction scenarios, namely $Q=3 H \xi \rho_{x}, Q=3 H \xi \rho_{c}$ and $Q=3 H \xi\left(\rho_{c}+\rho_{x}\right)$. Similarly to model IDE1 above, for the present model IDE2 we can see that for $z \rightarrow 0$ all curves tend to a non-zero value, which implies that the coincidence problem is alleviated. Additionally, although at low redshifts all models present similar behavior, at high redshifts the sign-changeable interaction models considered here are distinguishable from the known interacting models of the literature. 
Lastly, concerning the perturbations, in this scenario they are determined by $(13)-(16)$, with

$$
\frac{\delta Q}{Q}=\frac{\alpha \delta_{c} \rho_{c}-\beta \delta_{x} \rho_{x}}{\alpha \rho_{c}-\beta \rho_{x}}+\frac{2 \theta+h^{\prime}}{6 \mathcal{H}} .
$$

\section{THE DATA AND METHODOLOGY}

In this section we briefly describe the observational datasets and the methodology we follow in order to constrain the aforementioned interaction models.

1. Cosmic microwave background $(\mathrm{CMB})$ radiation: We consider the CMB data from Planck 2015 measurements [91, 92, and in particular we use the high- $\ell$ and low- $\ell$ temperature and polarization data from 91,92 .

2. Baryon acoustic oscillation (BAO): We use data from BAO distance measurements from the following sources. Data from 6dF Galaxy Survey (6dFGS) (redshift measurement at $z_{\text {eff }}=0.106$ ) 94, data from Main Galaxy Sample of Data Release 7 of Sloan Digital Sky Survey (SDSS-MGS) $\left(z_{\text {eff }}=0.15\right)$ [95], and data from CMASS and LOWZ samples of the latest Data Release 12 (DR12) of the Baryon Oscillation Spectroscopic Survey $(\mathrm{BOSS})\left(z_{\mathrm{eff}}=0.57\right)$ 96 and $\left(z_{\mathrm{eff}}=0.32\right)$ 96.

3. Supernovae Type Ia (SNIa): We use the most latest compilation of SNIa, consisting of 1048 data points spanned over the redshift interval $z \in[0.01,2.3]$ known as the Pantheon sample 93.

4. Cosmic Chronometers (CC): We use the Hubble parameter measurements from the cosmic chronometers. The total number of data is 30 and the measurements are spanned over the redshift interval $0<z<2$ [97. For technical details we further refer to $[97$.

In order to perform the analysis and extract the observational constraints, we use the Markov chain Monte Carlo package cosmomc 98, 99] where a convergence diagnostic by Gelman-Rubin is included 100, which in addition supports the Planck 2015 likelihood code $\left[92{ }^{1}\right.$. In the case of IDE1 model we have the eight-dimensional parameter space: $\mathcal{P}_{1} \equiv\left\{\Omega_{b} h^{2}, \Omega_{c} h^{2}, 100 \theta_{M C}, \tau, w_{x}, n_{s}, \log \left[10^{10} A_{S}\right], \xi\right\}$, while for IVS1 we have one parameter less since $w_{x}=-1$. For IDE2 we have the nine-dimensional parameter space

${ }^{1}$ See the publicly available code at http://cosmologist.info/ cosmomc/ Note that although Planck 2018 cosmological parameters 101] are now available, the Planck 2018 likelihoods are still not available publicly, and thus we use the Planck 2015 ones.
$\mathcal{P}_{2} \equiv\left\{\Omega_{b} h^{2}, \Omega_{c} h^{2}, 100 \theta_{M C}, \tau, w_{x}, n_{s}, \log \left[10^{10} A_{S}\right], \alpha, \beta\right\}$, and similarly for IVS2 we have one parameter less. In the above expressions $\Omega_{b} h^{2}$ is the physical baryons density, $\Omega_{c} h^{2}$ is the cold dark matter density, $100 \theta_{M C}$ is the ratio of sound horizon to the angular diameter distance, $\tau$ is the optical depth, $w_{x}$ is the equation-of-state parameter for dark energy, $n_{s}$ is the scalar spectral index, and $A_{S}$ is the amplitude of the initial power spectrum. The remaining parameters in $\mathcal{P}_{1}$ and $\mathcal{P}_{2}$, namely $\xi, \alpha$ and $\beta$ are the coupling parameters for the interaction models. Fi-

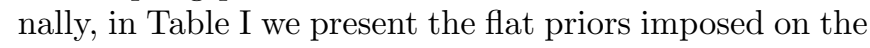
free parameters of the prescribed interacting scenarios.

\begin{tabular}{|c|c|}
\hline Parameter & Prior \\
\hline$\Omega_{b} h^{2}$ & {$[0.005,0.1]$} \\
$\Omega_{c} h^{2}$ & {$[0.01,0.99]$} \\
$\tau$ & {$[0.01,0.8]$} \\
$n_{s}$ & {$[0.5,1.5]$} \\
$\log \left[10^{10} A_{s}\right]$ & {$[2.4,4]$} \\
$100 \theta_{M C}$ & {$[0.5,10]$} \\
$w_{x}$ & {$[-2,0]$} \\
$\xi$ & {$[-1,0]$} \\
$\alpha$ & {$[-1,0]$} \\
$\beta$ & {$[-1,0]$} \\
\hline
\end{tabular}

TABLE I: The flat priors on the cosmological parameters used in the present analyses.

\section{OBSERVATIONAL CONSTRAINTS}

In this section we provide the observational constraints on the sign-changeable scenarios and we discuss their consequences. In order to acquire a complete picture we consider three different combinations of the observational datasets described above, namely $\mathrm{CMB}+\mathrm{BAO}, \mathrm{CMB}+\mathrm{BAO}+$ Pantheon and $\mathrm{CMB}+\mathrm{BAO}+$ Pantheon $+\mathrm{CC}$.

\section{A. Interaction function $Q=3 H \xi\left(\rho_{c}-\rho_{x}\right)$}

The observational summary for IDE1 model is presented in Table II. Additionally, in Fig. 7] we provide the corresponding $2 \mathrm{D}$ contour plots of various parameters at $1 \sigma$ and $2 \sigma$ confidence level (CL), alongside the 1D marginalized posterior distribution. From Table II we can notice that the addition of Pantheon or Pantheon $+\mathrm{CC}$ to the observational combination 


\begin{tabular}{|c|c|c|c|}
\hline Parameters & $\mathrm{CMB}+\mathrm{BAO}$ & $\mathrm{CMB}+\mathrm{BAO}+$ Pantheon & $\mathrm{CMB}+\mathrm{BAO}+$ Pantheon $+\mathrm{CC}$ \\
\hline$\Omega_{c} h^{2}$ & $0.1198_{-0.0013-0.0026}^{+0.0013+0.0024}$ & $0.1196_{-0.0012-0.0023}^{+0.0012+0.0024}$ & $0.1196_{-0.0013-0.0023}^{+0.0011+0.0024}$ \\
\hline$\Omega_{b} h^{2}$ & $0.02231_{-0.00016-0.00029}^{+0.00016+0.00032}$ & $0.02231_{-0.00015-0.00031}^{+0.00016+0.00029}$ & $0.02230_{-0.00016-0.00030}^{+0.00015}$ \\
\hline $100 \theta_{M C}$ & $1.04051_{-0.00031-0.00066}^{+0.00032+0.00070}$ & $1.04054_{-0.00032-0.00064}^{+0.00033+0.00064}$ & $1.04053_{-0.00030-0.00058}^{+0.00031+0.00057}$ \\
\hline$\tau$ & $0.082_{-0.017-0.032}^{+0.017+0.032}$ & $0.084_{-0.016-0.031}^{+0.017+0.030}$ & $0.082_{-0.017-0.034}^{+0.016+0.032}$ \\
\hline$n_{s}$ & $0.9741_{-0.0041-0.0080}^{+0.0041+0.0078}$ & $0.9741_{-0.0046-0.0080}^{+0.0040+0.0080}$ & $0.9742_{-0.0038-0.0074}^{+0.0037+0.0073}$ \\
\hline $\ln \left(10^{10} A_{s}\right)$ & $3.104_{-0.034-0.063}^{+0.034+0.066}$ & $3.108_{-0.031-0.061}^{+0.033+0.065}$ & $3.105_{-0.033-0.066}^{+0.032+0.063}$ \\
\hline$w_{x}$ & $-1.0818_{-0.0328-0.0916}^{+0.0634+0.0807}$ & $-1.0529_{-0.0206-0.0555}^{+0.0352+0.0483}$ & $-1.0545_{-0.0204-0.0545}^{+0.0342+0.0482}$ \\
\hline$\xi$ & $-0.00014_{-0.00004-0.00019}^{+0.00014+0.00014}$ & $-0.00013_{-0.00003-0.00020}^{+0.00013+0.00013}$ & $-0.00013_{-0.00003-0.00020}^{+0.00013+0.00013}$ \\
\hline$\Omega_{m 0}$ & $0.299_{-0.010-0.020}^{+0.011+0.018}$ & $0.305_{-0.008-0.013}^{+0.007+0.014}$ & $0.305_{-0.007-0.014}^{+0.007+0.014}$ \\
\hline$\sigma_{8}$ & $0.845_{-0.017-0.034}^{+0.017+0.036}$ & $0.840_{-0.014-0.027}^{+0.015+0.027}$ & $0.839_{-0.014-0.028}^{+0.014+0.030}$ \\
\hline$H_{0}$ & $69.12_{-1.39-2.11}^{+0.93+2.40}$ & $68.39_{-0.69-1.37}^{+0.68+1.39}$ & $68.43_{-0.76-1.39}^{+0.67+1.44}$ \\
\hline
\end{tabular}

TABLE II: Summary of the $68 \%(1 \sigma)$ and $95 \%(2 \sigma)$ confidence-level (CL) constraints on the interaction model IDE1 of 18 , using various combinations of the observational data sets. Here, $\Omega_{m 0}$ denotes the present value of $\Omega_{m}=\Omega_{b}+\Omega_{c}$ and $H_{0}$ is in the units of $\mathrm{km} / \mathrm{s} / \mathrm{Mpc}$.

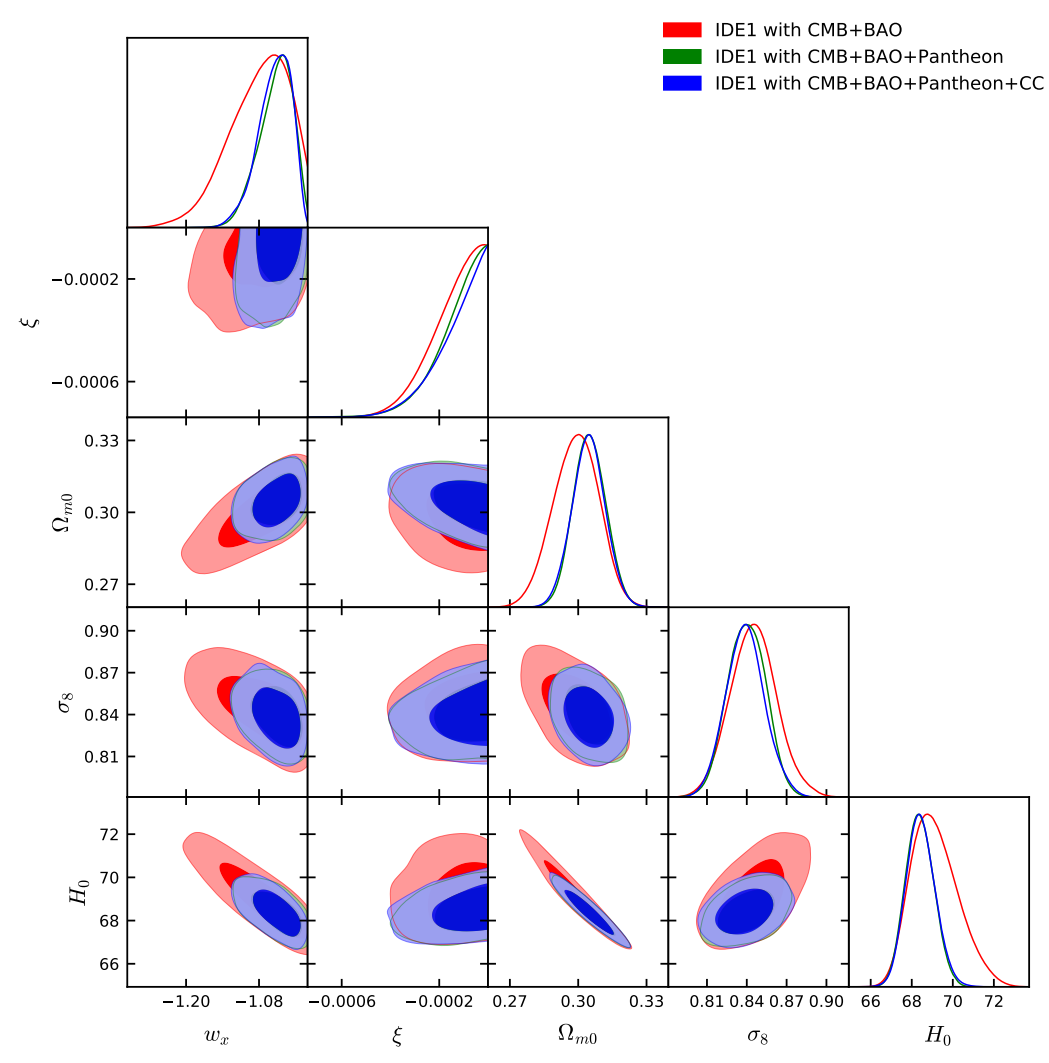

FIG. 7: The $1 \sigma$ and $2 \sigma$ CL contour plots for several combinations of various quantities and using various combinations of the observational data sets, for the interaction model IDE1 of (18), and the corresponding 1-dimensional (1D) marginalized posterior distributions. Here, $\Omega_{m 0}$ denotes the present value of $\Omega_{m}=\Omega_{b}+\Omega_{c}$ and $H_{0}$ is in the units of $\mathrm{km} / \mathrm{s} / \mathrm{Mpc}$.

$\mathrm{CMB}+\mathrm{BAO}$, slightly improves the constraints by reducing their error bars, nevertheless the improvement is not significant. Additionally, from Fig. 7 we can see that the parameters $\left(H_{0}, w_{x}\right)$ and $\left(H_{0}, \Omega_{m 0}\right)$ are negatively correlated to each other.

Concerning the dark energy equation-of-state parameter $w_{x}$, from Fig. 7 we infer that a phantom nature of $w_{x}$ is clearly preferred at more than $2 \sigma$ CL. Additionally, concerning the coupling $\xi$ and the dark energy equation-of-state parameter $w_{x}$, from Table II it is clearly observed that the evidence for an interaction is small $\left(\xi=-0.00014_{-0.00004}^{+0.00014}\right.$ at $1 \sigma$ for $\mathrm{CMB}+\mathrm{BAO}, \xi=$ $-0.00013_{-0.00003}^{+0.00013}$ at $1 \sigma$ for $\mathrm{CMB}+\mathrm{BAO}+$ Pantheon, $\xi=$ $-0.00013_{-0.00003}^{+0.00013}$ at $1 \sigma$ for $\mathrm{CMB}+\mathrm{BAO}+$ Pantheon $\left.+\mathrm{CC}\right)$. 


\begin{tabular}{|c|c|c|c|}
\hline Parameters & $\mathrm{CMB}+\mathrm{BAO}$ & $\mathrm{CMB}+\mathrm{BAO}+$ Pantheon & $\mathrm{CMB}+\mathrm{BAO}+$ Pantheon $+\mathrm{CC}$ \\
\hline$\Omega_{c} h^{2}$ & $0.1187_{-0.0010-0.0020}^{+0.0011+0.0020}$ & $0.1184_{-0.0010-0.0019}^{+0.0010+0.0019}$ & $0.1184_{-0.0009-0.0020}^{+0.0009+0.0019}$ \\
\hline$\Omega_{b} h^{2}$ & $0.02234_{-0.00015-0.00028}^{+0.00014+0.00029}$ & $0.02236_{-0.00015-0.00028}^{+0.00015+0.00028}$ & $0.02235_{-0.00015-0.00030}^{+0.00014+0.00030}$ \\
\hline $100 \theta_{M C}$ & $1.04064_{-0.00030-0.00059}^{+0.00030+0.00060}$ & $1.04066_{-0.00035-0.00059}^{+0.00030+0.00067}$ & $1.04066_{-0.00032-0.000060}^{+0.00032+0.0061}$ \\
\hline$\tau$ & $0.088_{-0.016-0.033}^{+0.016+0.031}$ & $0.089_{-0.017-0.032}^{+0.017+0.032}$ & $0.087_{-0.017-0.031}^{+0.016+0.032}$ \\
\hline$n_{s}$ & $0.9767_{-0.0037-0.0077}^{+0.0038+0.0077}$ & $0.9773_{-0.0038-0.0070}^{+0.0035+0.0070}$ & $0.9773_{-0.0038-0.0072}^{+0.0035+0.0076}$ \\
\hline $\ln \left(10^{10} A_{s}\right)$ & $3.115_{-0.031-0.064}^{+0.032+0.062}$ & $3.117_{-0.034-0.065}^{+0.033+0.063}$ & $3.112_{-0.034-0.061}^{+0.033+0.064}$ \\
\hline$\xi$ & $-0.000089_{-0.000018-0.000151}^{+0.000089+0.000089}$ & $-0.000080_{-0.000016-0.000080}^{+0.000080+0}$ & $-0.000078_{-0.000018-0.000129}^{+0.000078+0.000078}$ \\
\hline$\Omega_{m 0}$ & $0.312_{-0.007-0.013}^{+0.007+0.014}$ & $0.310_{-0.006-0.013}^{+0.006+0.013}$ & $0.311_{-0.006-0.012}^{+0.006+0.012}$ \\
\hline$\sigma_{8}$ & $0.825_{-0.014-0.027}^{+0.014+0.026}$ & $0.825_{-0.014-0.028}^{+0.014+0.027}$ & $0.824_{-0.014-0.026}^{+0.014+0.026}$ \\
\hline$H_{0}$ & $67.36_{-0.52-1.03}^{+0.52+1.02}$ & $67.50_{-0.48-0.98}^{+0.48+0.97}$ & $67.49_{-0.46-0.91}^{+0.46+0.95}$ \\
\hline
\end{tabular}

TABLE III: Summary of the $1 \sigma$ and $2 \sigma$ CL constraints on the interaction model IVS1, using various combinations of the observational data sets. Here, $\Omega_{m 0}$ denotes the present value of $\Omega_{m}=\Omega_{b}+\Omega_{c}$ and $H_{0}$ is in the units of $\mathrm{km} / \mathrm{s} / \mathrm{Mpc}$.
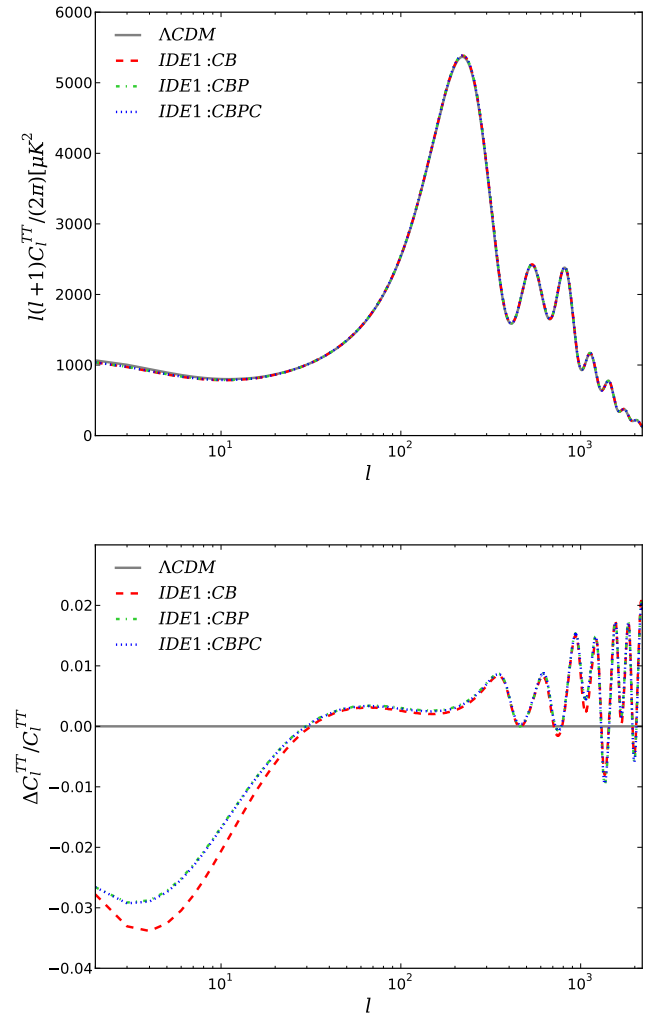

FIG. 8: The temperature anisotropy in the CMB TT spectra for the interaction model IDE1 of (18), considering three different combinations of the observational datasets, namely $C B$ (= $C M B+B A O), C B P(=C M B+B A O+$ Pantheon) and $C B P C(=C M B+B A O+$ Pantheon $+C C)$, as well as the curve for $\Lambda C D M$ paradigm (upper graph), and the corresponding residual plot with reference to $\Lambda$ CDM scenario (lower graph).

Hence, within $1 \sigma$ the non-interacting cosmology is allowed. In summary, our observational confrontation shows that this interaction model at the background level essentially mimicks a $w_{x}$ CDM-type cosmology with $w_{x}<-1$ at more than $2 \sigma$ CL.
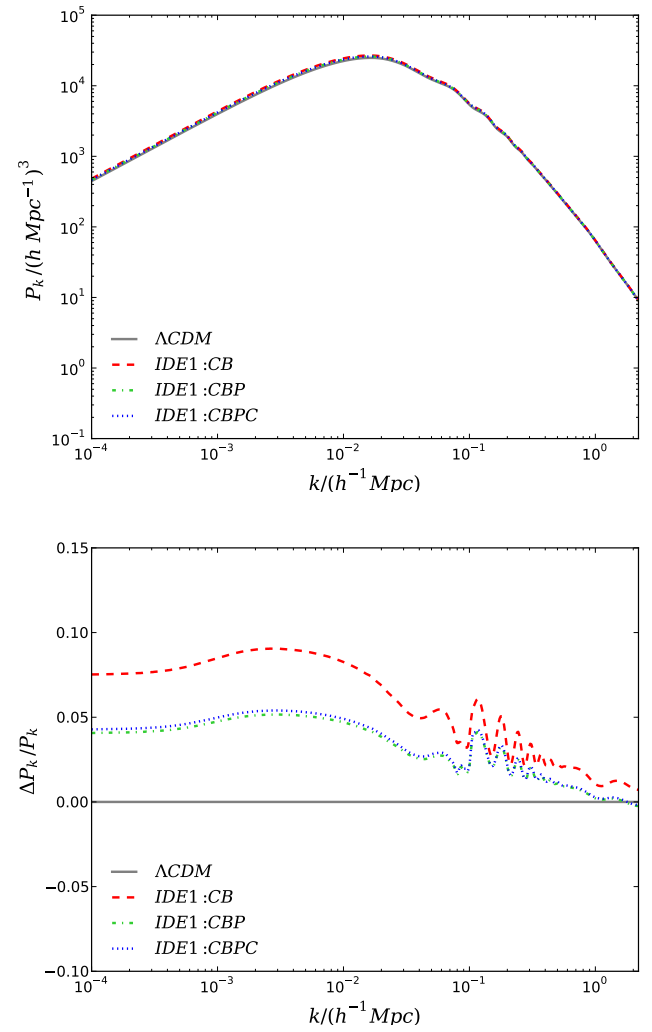

FIG. 9: The matter power spectra for the interaction model IDE1 of (18), considering three different combinations of the observational datasets, namely $C B$ (= $C M B+B A O), C B P(=C M B+B A O+$ Pantheon) and $C B P C$ (= $C M B+B A O+$ Pantheon $+C C)$, as well as the curve for $\Lambda C D M$ paradigm (upper graph), and the corresponding residual plot with reference to $\Lambda$ CDM scenario (lower graph).

We now proceed by examining the tensions on the two main parameters, namely $H_{0}$ and $\sigma_{8}$. As we observe from both Table II and Fig. 7 (specifically from the posterior distribution of $H_{0}$ which is the extreme right plot of the bottom panel), $H_{0}$ acquires slightly higher values 


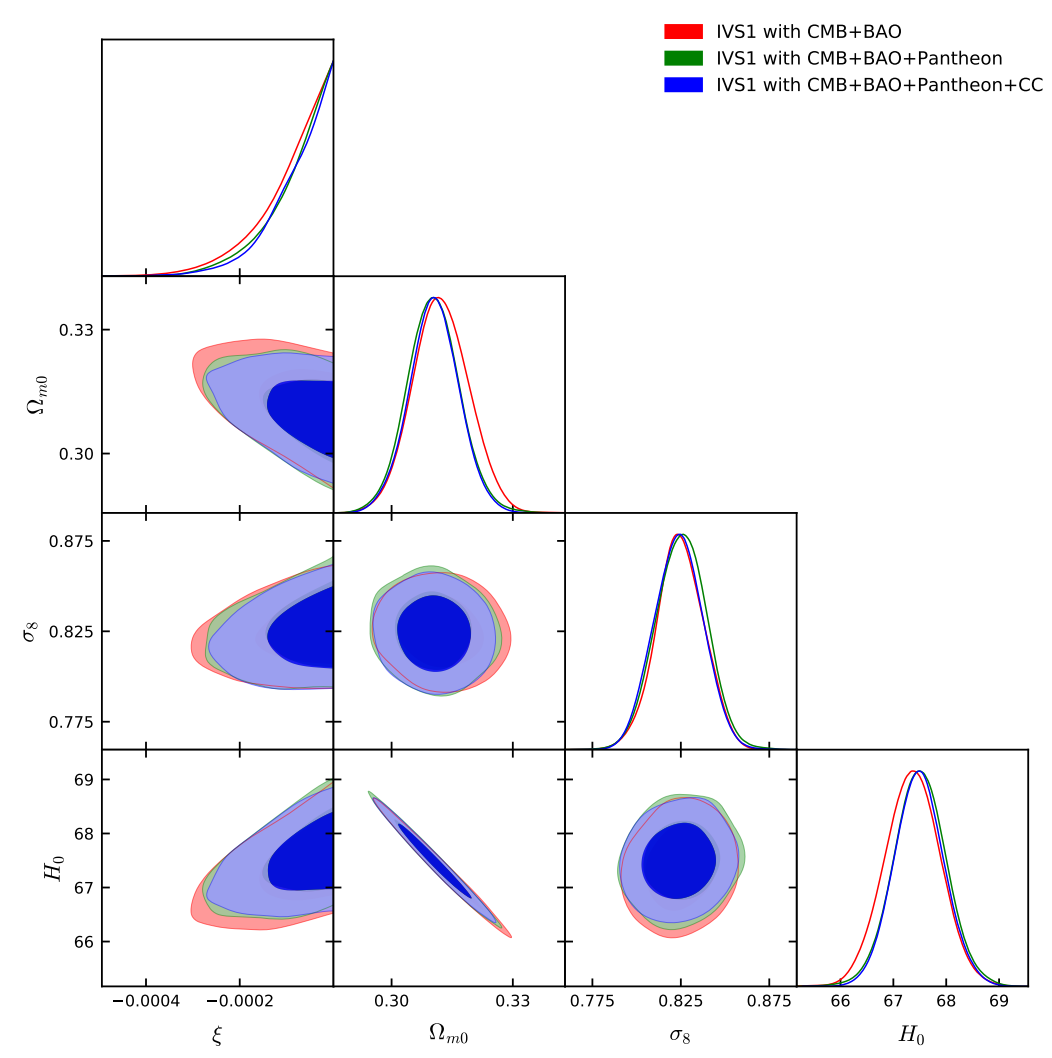

FIG. 10: The $1 \sigma$ and $2 \sigma$ CL contour plots for several combinations of various quantities and using various combinations of the observational data sets, for the interaction model IVS1, and the corresponding $1 D$ marginalized posterior distributions. Here, $\Omega_{m 0}$ denotes the present value of $\Omega_{m}=\Omega_{b}+\Omega_{c}$ and $H_{0}$ is in the units of $\mathrm{km} / \mathrm{s} / \mathrm{Mpc}$.

compared to Planck [1, with slightly higher error bars. Although the local estimation of the Hubble constant obtained by Riess et al. 107, i.e. $H_{0}=73.24 \pm 1.74$, is certainly greater than the estimated mean values of $H_{0}$ for this interaction model, due to the increased error bars the tension is reduced to the level of $2 \sigma$ CL. Thus, the interaction between the dark components provides a way to reduce the tension from $>3 \sigma$ CL. Nevertheless, concerning $\sigma_{8}$ tension we deduce that the present interaction model is not able to alleviate it.

Let us now examine the effect of the interaction on the large scale observables, and mainly on the CMB TT and matter power spectra. In the upper graph of Fig. 8 we show how the interaction affects the CMB TT spectra, considering the constraints on the parameters extracted from all observational datasets, namely $\mathrm{CMB}+\mathrm{BAO}, \mathrm{CMB}+\mathrm{BAO}+$ Pantheon and $\mathrm{CMB}+\mathrm{BAO}+$ Pantheon $+\mathrm{CC}$, in which for completeness we add the non-interacting case of $\Lambda \mathrm{CDM}$ cosmology. From this graph it is hard to distinguish the interacting case from $\Lambda \mathrm{CDM}$ scenario. However, in the lower graph of Fig. 8 we depict the corresponding residual plot (with reference to $\Lambda$ CDM model), and one can indeed trace a distinction between interacting and noninteracting cosmologies, mainly in the lower multipoles. Similarly, we investigate the effects of the interaction through the matter power spectra presented in Fig. 9. Although in the upper graph the distinction between interacting and non-interacting cosmologies cannot be observed, in the lower graph the deviation from the noninteracting $\Lambda \mathrm{CDM}$ cosmology is clear. This is one of the main results of the present work.

We close the analysis of this model by focusing on the case where $w_{x}=-1$, thus $w_{x}$ is not a free parameter and is fixed to the cosmological constant value, namely we examine the interacting vacuum scenario. In this case we summarize the results in Table III] and in Fig. 10 we present the corresponding contour plots. In this model the case of no-interaction seems to be favored, while $H_{0}$ acquires smaller values, and therefore the $H_{0}$ tension is not alleviated. Concerning $\sigma_{8}$ tension we deduce that it cannot be released either. Finally, at large scales this specific interacting scenario does not return different results in the CMB TT and matter power spectra comparing to $\Lambda$ CDM cosmology, and therefore we do not explicitly present the corresponding plots. 


\begin{tabular}{|c|c|c|c|}
\hline Parameters & $\mathrm{CMB}+\mathrm{BAO}$ & $\mathrm{CMB}+\mathrm{BAO}+$ Pantheon & $\mathrm{CMB}+\mathrm{BAO}+$ Pantheon+CC \\
\hline$\Omega_{c} h^{2}$ & $0.1127_{-0.0037-0.0133}^{+0.0080+0.0099}$ & $0.1149_{-0.0025-0.0078}^{+0.0048+0.0066}$ & $0.1145_{-0.0029-0.0078}^{+0.0051+0.0069}$ \\
\hline$\Omega_{b} h^{2}$ & $0.02229_{-0.00015-0.00029}^{+0.00015+0.00032}$ & $0.02229_{-0.00015-0.00031}^{+0.00015+0.00031}$ & $0.02230_{-0.00014-0.00029}^{+0.00014+0.00031}$ \\
\hline $100 \theta_{M C}$ & $1.04090_{-0.00052-0.00093}^{+0.00042+0.00097}$ & $1.04081_{-0.00036-0.00070}^{+0.00036+0.00071}$ & $1.04083_{-0.00040-0.00070}^{+0.00036+0.00077}$ \\
\hline$\tau$ & $0.079_{-0.017-0.033}^{+0.017+0.032}$ & $0.081_{-0.017-0.034}^{+0.017+0.032}$ & $0.081_{-0.017-0.033}^{+0.017+0.032}$ \\
\hline$n_{s}$ & $0.9731_{-0.0037-0.0075}^{+0.0037+0.0073}$ & $0.9738_{-0.0041-0.0074}^{+0.0038+0.0080}$ & $0.9736_{-0.0044-0.0079}^{+0.0038+0.0081}$ \\
\hline $\ln \left(10^{10} A_{s}\right)$ & $3.101_{-0.032-0.065}^{+0.032+0.062}$ & $3.104_{-0.032-0.067}^{+0.033+0.062}$ & $3.105_{-0.033-0.066}^{+0.033+0.063}$ \\
\hline$w_{x}$ & $-1.0931_{-0.0374-0.0865}^{+0.0529+0.0777}$ & $-1.0549_{-0.0181-0.0517}^{+0.0320+0.0455}$ & $-1.0536_{-0.0186-0.0496}^{+0.0290+0.0449}$ \\
\hline$\alpha$ & $-0.00015_{-0.00004-0.00019}^{+0.00015+0.00015}$ & $-0.00014_{-0.00003-0.00021}^{+0.00014+0.00014}$ & $-0.00014_{-0.00003-0.00020}^{+0.00014+0.00014}$ \\
\hline$\beta$ & $-0.02444_{-0.00681-0.04197}^{+0.02444+0.02444}$ & $-0.01649_{-0.00394-0.02375}^{+0.01649+0.01649}$ & $-0.01783_{-0.00529-0.02396}^{+0.01783+0.01783}$ \\
\hline$\Omega_{m 0}$ & $0.279_{-0.015-0.039}^{+0.023+0.034}$ & $0.292_{-0.010-0.024}^{+0.013+0.023}$ & $0.291_{-0.011-0.026}^{+0.014+0.023}$ \\
\hline$\sigma_{8}$ & $0.906_{-0.072-0.093}^{+0.032+0.122}$ & $0.875_{-0.040-0.061}^{+0.023+0.069}$ & $0.879_{-0.043-0.063}^{+0.025+0.072}$ \\
\hline$H_{0}$ & $69.81_{-1.39-2.40}^{+1.18+2.51}$ & $68.68_{-0.73-1.41}^{+0.73+1.41}$ & $68.72_{-0.80-1.37}^{+0.70+1.47}$ \\
\hline
\end{tabular}

TABLE IV: Summary of the $1 \sigma$ and $2 \sigma$ CL constraints on the interaction model IDE2 of (22), using various combinations of the observational data sets. Here, $\Omega_{m 0}$ denotes the present value of $\Omega_{m}=\Omega_{b}+\Omega_{c}$ and $H_{0}$ is in the units of km/s/Mpc.

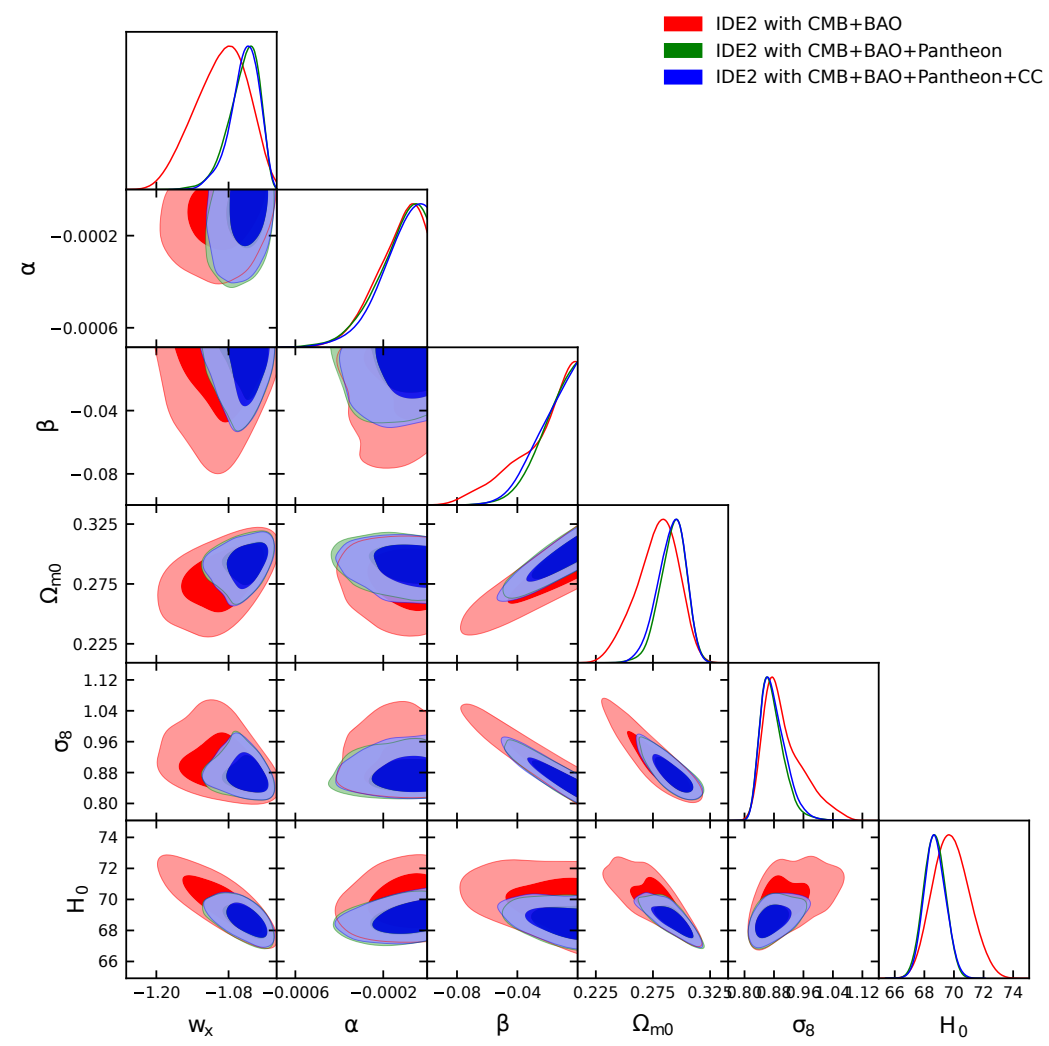

FIG. 11: The $1 \sigma$ and $2 \sigma$ CL contour plots for several combinations of various quantities and using various combinations of the observational data sets, for the interaction model IDE2 (22), and the corresponding $1 D$ marginalized posterior distributions. Here, $\Omega_{m 0}$ denotes the present value of $\Omega_{m}=\Omega_{b}+\Omega_{c}$ and $H_{0}$ is in the units of $\mathrm{km} / \mathrm{s} / \mathrm{Mpc}$.

\section{B. Interaction function $Q=3 H\left(\alpha \rho_{c}-\beta \rho_{x}\right)$}

The observational summary of model IDE2 is shown in Table IV, while the corresponding 2D contour plots are presented in Fig. 11. Similarly to model IDE1, one can notice that the addition of Pantheon or Pantheon+CC to the combined analysis $\mathrm{CMB}+\mathrm{BAO}$, improves the pa- rameters space only slightly. Moreover, from Fig. 11 we can see that the parameters $\left(H_{0}, w_{x}\right)$ and $\left(H_{0}, \Omega_{m 0}\right)$ are negatively correlated to each other.

Concerning the coupling parameters $\alpha$ and $\beta$, our analysis shows that the zero values are allowed within $1 \sigma$. Additionally, the dark-energy equation-of-state parameter prefers the phantom regime for all datasets, namely 

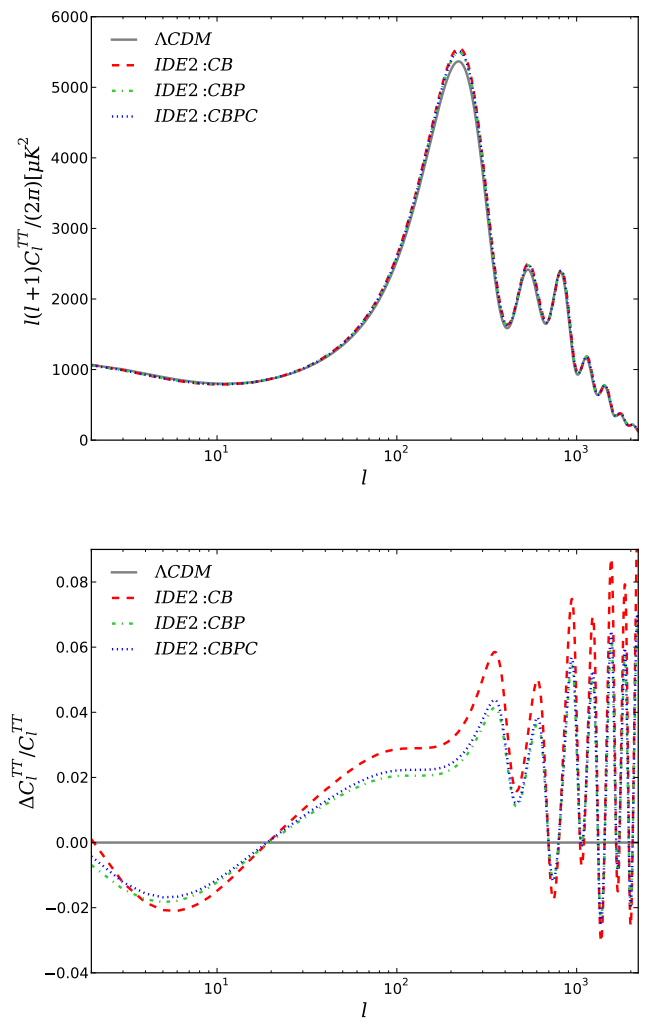

FIG. 12: The temperature anisotropy in the CMB TT spectra for the interaction model IDE2 of (22), considering three different combinations of the observational datasets, namely $C B$ (= $C M B+B A O), C B P(=C M B+B A O+$ Pantheon) and $C B P C(=C M B+B A O+$ Pantheon $+C C)$, as well as the curve for $\Lambda C D M$ paradigm (upper graph), and the corresponding residual plot with reference to $\Lambda$ CDM scenario (lower graph).

we see that $w_{x}<-1$ at more than $2 \sigma$. Furthermore, similarly to IDE1 model, in the present IDE2 scenario we also find that the estimations of $H_{0}$ are slightly higher compared to the $\Lambda$ CDM-based Planck estimation [1], and due to the higher error bars on $H_{0}$ the relevant tension can be slightly reconciled due to the interaction. However, concerning $\sigma_{8}$ we see that the tension is not released.

We proceed by investigating the effect of the interaction on the CMB TT and matter power spectra. In the upper graph of Fig. 12 we depict the CMB TT spectra considering the constraints on the parameters extracted from all observational datasets, namely $\mathrm{CMB}+\mathrm{BAO}, \mathrm{CMB}+\mathrm{BAO}+$ Pantheon, and $\mathrm{CMB}+\mathrm{BAO}+$ Pantheon $+\mathrm{CC}$, in which for completeness we add the non-interacting case of $\Lambda \mathrm{CDM}$ cosmology. Moreover, in the lower graph of Fig. 12 we present the corresponding residual plot (with reference to $\Lambda \mathrm{CDM}$ model). As we observe, this interaction model is distinguished from the non-interacting $\Lambda \mathrm{CDM}$ cosmology at both lower and higher multipoles. We further investigate the effects of the interaction on the matter power spectra, depicted in Fig. 13. Although in the upper graph the
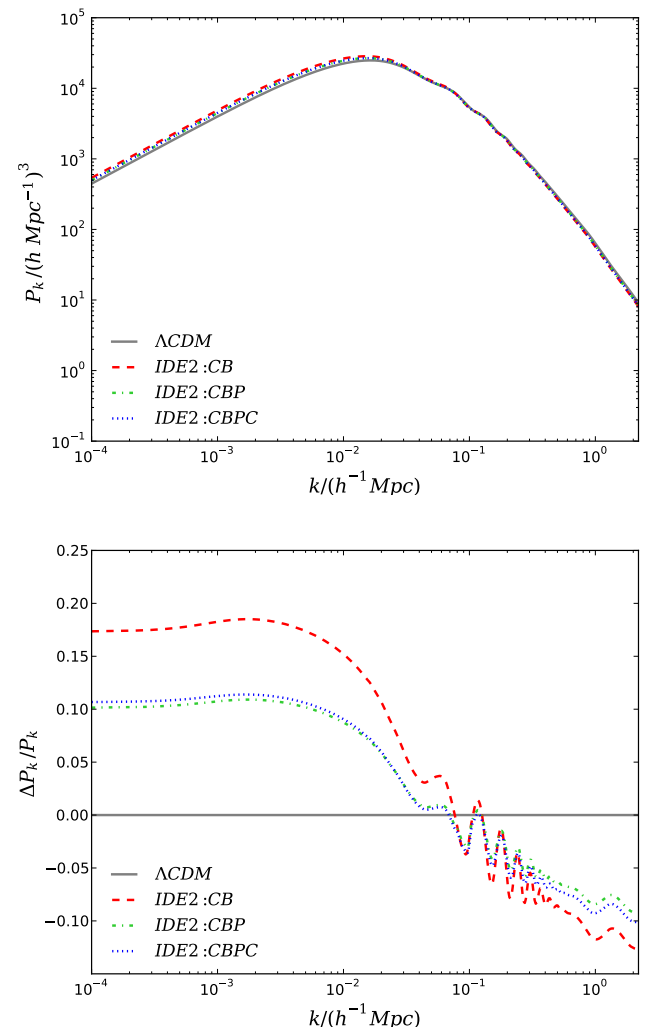

FIG. 13: The matter power spectra for the interaction model IDE2 of (22), considering three different combinations of the observational datasets, namely $C B$ (= $C M B+B A O), C B P(=C M B+B A O+$ Pantheon) and $C B P C$ $(=C M B+B A O+$ Pantheon $+C C)$, as well as the curve for $\Lambda C D M$ paradigm (upper graph), and the corresponding residual plot with reference to $\Lambda C D M$ scenario (lower graph).

distinction between the interacting and non-interacting cosmologies cannot be observed, in the lower graph the deviation from the non-interacting $\Lambda \mathrm{CDM}$ cosmology is clear even for the small values of the coupling parameters $\alpha, \beta$ that were obtained from the three different observational datasets. This is one of the main results of the present work.

We proceed by analyzing the case $w_{x}=-1$, namely the interacting vacuum scenario. The results of the analyses are presented in Table $\mathrm{V}$, while in Fig. 14 we depict the corresponding contour plots. From these we deduce that the coupling parameter $\alpha$ acquires the zero value within $1 \sigma$ CL irrespectively of the datasets, while $\beta$ has a tendency towards non-zero values nevertheless the value zero is allowed within $1 \sigma$ except for the final combination $\mathrm{CMB}+\mathrm{BAO}+$ Pantheon $+\mathrm{CC}$. Additionally, concerning $H_{0}$, for $\mathrm{CMB}+\mathrm{BAO}+$ Pantheon and $\mathrm{CMB}+\mathrm{BAO}+$ Pantheon $+\mathrm{CC}$ datasets we find that its estimations are relatively high compared to $\Lambda \mathrm{CDM}$ based Planck estimation [1, while the error bars on $H_{0}$ 


\begin{tabular}{|c|c|c|c|}
\hline Parameters & $\mathrm{CMB}+\mathrm{BAO}$ & $\mathrm{CMB}+\mathrm{BAO}+$ Pantheon & $\mathrm{CMB}+\mathrm{BAO}+$ Pantheon $+\mathrm{CC}$ \\
\hline$\Omega_{c} h^{2}$ & $0.1141_{-0.0020-0.0085}^{+0.0048+0.0069}$ & $0.1085_{-0.0044-0.0159}^{+0.0101+0.0126}$ & $0.1055_{-0.0053-0.0181}^{+0.0111+0.0149}$ \\
\hline$\Omega_{b} h^{2}$ & $0.02234_{-0.00013-0.00026}^{+0.00013}$ & $0.02233_{-0.00015-0.00030}^{+0.00016+0.00030}$ & $0.02231_{-0.00015-0.00029}^{+0.00014+0.00030}$ \\
\hline $100 \theta_{M C}$ & $1.04088_{-0.00035+0.00086}^{+0.00035+0.0008}$ & $1.04119_{-0.00044+0.00107}^{+0.00048}$ & $1.04135_{-0.000069-0.00123}^{+0.00045+0.001}$ \\
\hline$\tau$ & $0.087_{-0.016-0.035}^{+0.018+0.033}$ & $0.085_{-0.017-0.032}^{+0.017+0.032}$ & $0.084_{-0.017-0.032}^{+0.017+0.032}$ \\
\hline$n_{s}$ & $0.9766_{-0.0037-.0080}^{+0.0036+0.0079}$ & $0.9758_{-0.0039-0.0076}^{+0.0040+0.0079}$ & $0.9753_{-0.0038-0.0076}^{+0.0038+0.0076}$ \\
\hline $\ln \left(10^{10} A_{s}\right)$ & $3.114_{-0.031-0.069}^{+0.036+0.062}$ & $3.111_{-0.032-0.062}^{+0.032+0.064}$ & $3.109_{-0.033-0.066}^{+0.034+0.064}$ \\
\hline$\alpha$ & $-0.000092_{-0.000019-0.000092}^{+0.000092}$ & $-0.000099_{-0.000023-0.000157}^{+0.000099+0.000099}$ & $-0.000094_{-0.000022-0.000147}^{+0.000094+0.000094}$ \\
\hline$\beta$ & $-0.01474_{-0.00223-0.02488}^{+0.01474+0.01474}$ & $-0.03330_{-0.00844-0.04914}^{+0.03330+0.03330}$ & $-0.04238_{-0.01469-0.05246}^{+0.03732+0.04238}$ \\
\hline$\Omega_{m 0}$ & $0.299_{-0.009-0.027}^{+0.014+0.025}$ & $0.284_{-0.015-0.042}^{+0.026+0.036}$ & $0.276_{-0.016-0.048}^{+0.029+0.042}$ \\
\hline$\sigma_{8}$ & $0.857_{-0.037-0.058}^{+0.019+0.068}$ & $0.902_{-0.084-0.106}^{+0.032+0.143}$ & $0.927_{-0.093-0.127}^{+0.039+0.163}$ \\
\hline$H_{0}$ & $67.71_{-0.57-1.15}^{+0.61+1.11}$ & $68.05_{-0.64-1.29}^{+0.67+1.24}$ & $68.24_{-0.80-1.30}^{+0.64+1.50}$ \\
\hline
\end{tabular}

TABLE V: Summary of the $1 \sigma$ and $2 \sigma$ CL constraints on the interaction model IVS2, using various combinations of the observational data sets. Here, $\Omega_{m 0}$ denotes the present value of $\Omega_{m}=\Omega_{b}+\Omega_{c}$ and $H_{0}$ is in the units of $\mathrm{km} / \mathrm{s} / \mathrm{Mpc}$.

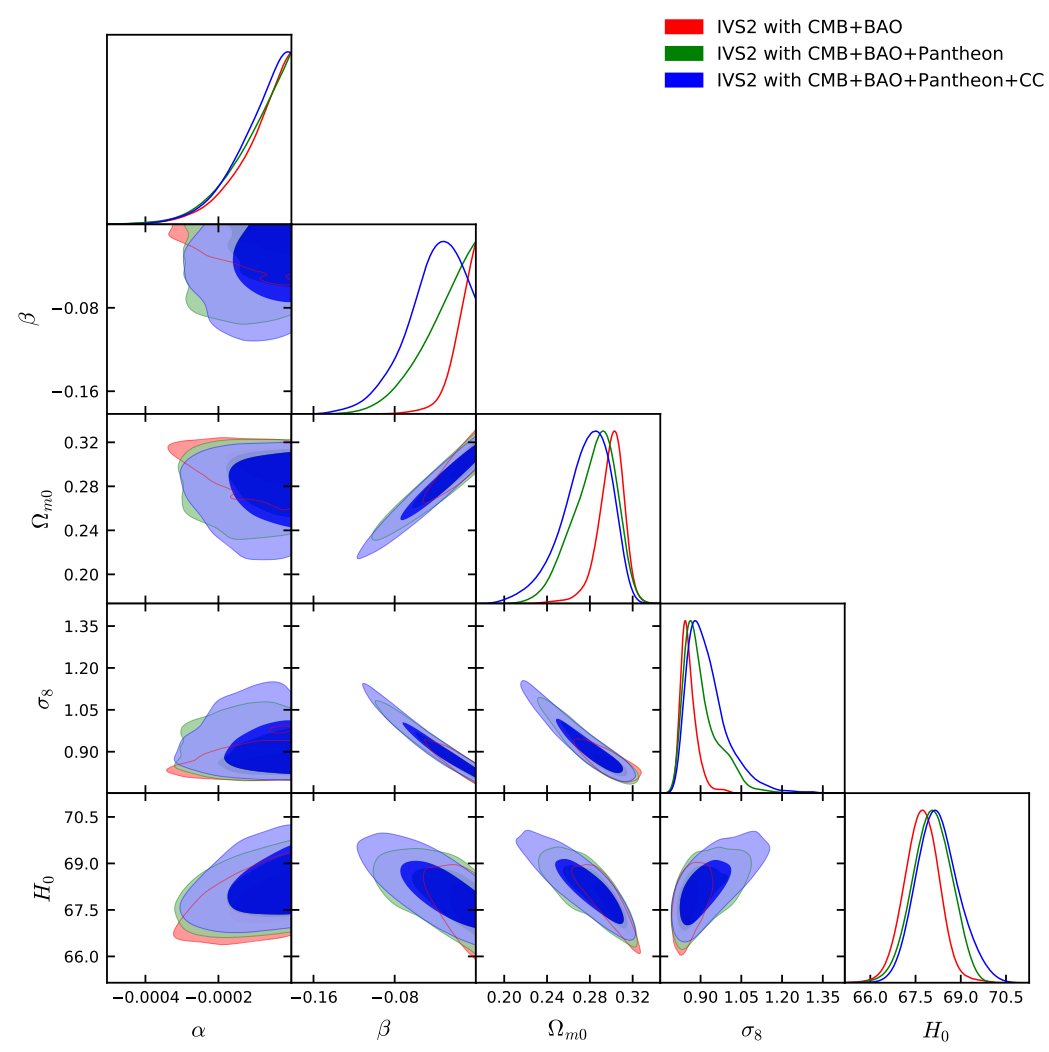

FIG. 14: The $1 \sigma$ and $2 \sigma$ CL contour plots for several combinations of various quantities and using various combinations of the observational data sets, for the interaction model IVS2, and the corresponding $1 D$ marginalized posterior distributions. Here, $\Omega_{m 0}$ denotes the present value of $\Omega_{m}=\Omega_{b}+\Omega_{c}$ and $H_{0}$ is in the units of $\mathrm{km} / \mathrm{s} / \mathrm{Mpc}$.

are also increased. Hence, this enables $H_{0}$ to acquire values close to its local estimation 107, and thus the tension on $H_{0}$ is weakly resolved. However, the $\sigma_{8}$ tension cannot be alleviated.

\section{Bayesian analysis}

We close the observational confrontation by presenting observational viabilities of the models using the Bayesian evidence. The Bayesian analysis is an important part of the cosmological model selection that quantifies the fitting results compared to a reference scenario. The com- 
putation of Bayesian evidence is performed with the code MCEvidence [104, 105] ${ }^{2}$, which directly computes the evidences of the model with respect to the reference $\Lambda \mathrm{CDM}$ scenario.

In Bayesian analysis one needs to calculate the posterior probability of the model parameters $\theta$, subject to a particular observational dataset $x$ and any prior information for the underlying model $M$. Recalling the Bayes theorem one can write that

$$
p(\theta \mid x, M)=\frac{p(x \mid \theta, M) \pi(\theta \mid M)}{p(x \mid M)},
$$

in which $p(x \mid \theta, M)$ is the likelihood function (depending on the model parameters $\theta$ with the given data set), and where $\pi(\theta \mid M)$ refers to the prior information. The quantity $p(x \mid M)$ is the Bayesian evidence. Given two models, namely $M_{i}$ and $M_{j}$, where $M_{i}$ is the model under investigation and $M_{j}$ is the reference model (here the $\Lambda \mathrm{CDM}$ scenario), the posterior probability is given by

$$
\frac{p\left(M_{i} \mid x\right)}{p\left(M_{j} \mid x\right)}=\frac{\pi\left(M_{i}\right)}{\pi\left(M_{j}\right)} \frac{p\left(x \mid M_{i}\right)}{p\left(x \mid M_{j}\right)}=\frac{\pi\left(M_{i}\right)}{\pi\left(M_{j}\right)} B_{i j}
$$

The quantity $B_{i j}=\frac{p\left(x \mid M_{i}\right)}{p\left(x \mid M_{j}\right)}$ is the Bayes factor of the considered model $M_{i}$ relative to the reference model $M_{j}$, and quantifies how the observational data support the model $M_{i}$ over $M_{j}$. In Table VI we show the corresponding classification following [103].

\begin{tabular}{cc}
\hline \hline $\ln B_{i j}$ & Strength of evidence for model $M_{i}$ \\
\hline $0 \leq \ln B_{i j}<1$ & Weak \\
$1 \leq \ln B_{i j}<3$ & Definite/Positive \\
$3 \leq \ln B_{i j}<5$ & Strong \\
$\ln B_{i j} \geq 5$ & Very strong \\
\hline \hline
\end{tabular}

TABLE VI: The Revised Jeffreys scale from [103 that quantifies the fitting efficiency of the investigated model $M_{i}$ comparing to the reference model $M_{j}$.

In Table VII we present the computed values of $\ln B_{i j}$ for all sign-changeable interacting scenarios considering all observational datasets. As we can see, the values of $\left|\ln B_{i j}\right|$ for IDE2 are greater than the values of $\left|\ln B_{i j}\right|$ for IDE1 and this is true for all datasets. This was expected since IDE2 scenario has one extra free parameter compared to IDE1. Similarly, the values of $\left|\ln B_{i j}\right|$ for model IVS2 are greater than those IVS1. Nevertheless, overall, $\Lambda \mathrm{CDM}$ cosmology is still favored over the present interacting models.

\footnotetext{
${ }^{2}$ See the freely available code in github.com/yabebalFantaye/MCEvidence
}

\begin{tabular}{ccc}
\hline \hline Dataset & Model & $\ln B_{i j}$ \\
\hline CB & IDE1 & -4.8 \\
CBP & IDE1 & -2.4 \\
CBPC & IDE1 & -2.9 \\
\hline CB & IVS1 & -3.9 \\
CBP & IVS1 & -2.9 \\
CBPC & IVS1 & -3.1 \\
\hline \hline CB & IDE2 & -6.6 \\
CBP & IDE2 & -3.1 \\
CBPC & IDE2 & -4.2 \\
\hline CB & IVS2 & -5.1 \\
CBP & IVS2 & -3.6 \\
CBPC & IVS2 & -4.0 \\
\hline \hline
\end{tabular}

TABLE VII: The values of $\ln B_{i j}$ for all interaction scenarios comparing to the reference paradigm of $\Lambda \mathrm{CDM}$, for all observational datasets. Here, $\mathrm{CB}=$ $\mathrm{CMB}+\mathrm{BAO}, \mathrm{CBP}=\mathrm{CMB}+\mathrm{BAO}+$ Pantheon and $\mathrm{CBPC}=$ $\mathrm{CMB}+\mathrm{BAO}+\mathrm{Pantheon}+\mathrm{CC}$. The negative values of $\ln B_{i j}$ imply that the $\Lambda \mathrm{CDM}$ paradigm is preferred over the interaction scenarios.

\section{LAWS OF THERMODYNAMICS IN SIGN-CHANGEABLE INTERACTION MODELS}

In this section we shall investigate the thermodynamical laws in a universe governed by sign-changeable interacting dark energy. In order to investigate the thermodynamical properties of a specific cosmological model one assumes that the universe is a thermodynamical system bounded by a cosmological horizon, and then he applies arguments from black hole thermodynamics [108, 109, 110]. In particular, one considers that the universe is bounded by the apparent horizon with radius $r_{h}=\left(H^{2}+k / a^{2}\right)^{-1 / 2}$ [11] which, for a spatially flat universe becomes the Hubble horizon $r_{h}=1 / H$. Hence, one can show that the first law of thermodynamics can lead to the first Friedmann equation [112.

We proceed by examining the validity of the generalized second law of thermodynamics, which states that the total entropy of the universe, namely the entropy of the various cosmological fluids plus the entropy of the horizon, should be a non-decreasing function of time [113. Concerning the entropy of the various fluids that constitute the universe one has $S_{r}+S_{b}+S_{c}+S_{x}$, where $S_{i}$ $(i=r, b, c, x)$ denotes the entropy of the $i$-th fluid. Thus, the first law of thermodynamics for each individual fluid becomes [109, 110, 111, 112, 114, 115, 116, 117, 118.

$$
\begin{aligned}
& T d S_{r}=d E_{r}+p_{r} d V, \\
& T d S_{b}=d E_{b}+p_{b} d V=d E_{b}, \\
& T d S_{c}=d E_{c}+p_{c} d V=d E_{c}, \\
& T d S_{x}=d E_{x}+p_{x} d V,
\end{aligned}
$$

where $V=4 \pi r_{h}^{3} / 3$ is the volume of the universe, $E_{i}$ 
stands for the internal energy of the $i$-th fluid given by $E_{i}=\frac{4}{3} \pi r_{h}^{3} \rho_{i}$, and $p_{i}$ is the corresponding pressure. Note that the various fluids are considered to have the same temperature. However, we mention that over the entire cosmic evolution the temperatures of different cosmic fluids are not the same [119], as they evolve differently. In particular, the temperatures of radiation and dark energy remain different from the horizon temperature for a long period of time, while for the non-relativistic matter its temperature becomes and remains equal with the horizon one. Nevertheless, if at some epoch in the universe evolution the horizon temperature comes close or become equal to the temperature of the dark energy sector, then they will remain the same for most of the expansion of the universe. Therefore, the assumption, that the temperatures of different cosmic fluids are the same, is not unjustified [119].

The entropy of the horizon is taken to be that of a black hole, namely $S_{h}=k_{B} \mathcal{A} /\left(4 l_{p l}^{2}\right)$, with $k_{B}$ the Boltzmann's constant, $\hbar$ the Planck's constant, and $l_{p l}=\left(\sqrt{\hbar G / c^{3}}\right)$ the Planck's length, however taking the area to be that corresponding to the horizon, i.e. $\mathcal{A}=4 \pi r_{h}^{2}$ [108, 109, 110. Therefore, in units where $\hbar=k_{B}=c=8 \pi G=1$, the horizon entropy 32 reduces to

$$
S_{h}=8 \pi^{2} r_{h}^{2}
$$

Moreover, concerning the temperature of the apparent horizon one can use the black hole result but with the apparent horizon instead of the black-hole one, namely $T_{h}=1 /\left(2 \pi r_{h}\right)$ [109, 110, 111, 112, 113, 114, 115, 116, 117, 118 .

Using all the above relations together with the assumption that the temperature of the fluids $T$ should be equal to $T_{h}$ one can find that

$$
\begin{aligned}
\dot{S} & =\dot{S}_{r}+\dot{S}_{b}+\dot{S}_{c}+\dot{S}_{x}+\dot{S}_{h} \\
& =4 \pi^{2} H r_{h}^{6}\left[\rho_{r}\left(1+w_{r}\right)+\rho_{b}+\rho_{c}+\left(1+w_{x}\right) \rho_{x}\right]^{2}
\end{aligned}
$$

where we have replaced the involved $\dot{r}_{h}=-\dot{H} / H^{2}$ using the Friedmann equations $\sqrt{2}, \sqrt{3}$. . As we can see, the total entropy is always a non-decreasing function, and thus the generalized second law of thermodynamics is satisfied. Hence, although the sign change of the interaction between dark matter and dark energy could lead to a local entropy decrease at the microscopic level, the total entropy of the universe plus the one of the horizon is always non-decreasing. The above relation for the first law of thermodynamics in interacting cosmology has been established earlier in 62 .

Now, let us examine whether the universe with signchangeable interaction in the dark sectors will result to thermodynamic equilibrium. As it is discussed extensively in the literature (see for instance [120, 121]), in order for this to be achieved one needs to have a total entropy whose first derivative is positive, while at late times its second derivative should be negative [122, and thus the entropy asymptotically tends towards a constant value. Using $x=\ln a$ as the independent variable the above require that $S^{\prime} \geq 0$ and $S^{\prime \prime}<0$, where $S=S_{r}+S_{b}+S_{c}+S_{x}+S_{h}$, with primes denoting differentiation with respect to $x$.

Equation (33) can be rewritten as

$$
S^{\prime}=\frac{16 \pi^{2}}{H^{4}}\left(H^{\prime}\right)^{2}
$$

and thus it leads to

$$
S^{\prime \prime}=2 S^{\prime}\left(\frac{H^{\prime \prime}}{H^{\prime}}-\frac{2 H^{\prime}}{H}\right)=2 S^{\prime}\left(\frac{h^{\prime \prime}}{h^{\prime}}-\frac{2 h^{\prime}}{h}\right)=2 S^{\prime} \Delta,
$$

where $h=H / H_{0}$ and $\Delta=\left(\frac{h^{\prime \prime}}{h^{\prime}}-\frac{2 h^{\prime}}{h}\right)$. In Fig. 15 we depict the evolution of $S^{\prime \prime}$ for both IDE1 and IDE2 models. As we observe, in both models at late times, namely at present epoch $(\ln a=0)$ and in the future, the total entropy is convex, i.e. $S^{\prime \prime}<0$, and hence the universe tends towards thermodynamic equilibrium. This can also be seen by the fact that asymptotically $S^{\prime \prime}$ goes to zero. These features hold also for the two models IVS1 and IVS2. Moreover, note that the individual curves are practically indistinguishable.

Hence, in summary, we conclude that for cosmological scenarios with sign changeable interaction functions the thermodynamical laws remain valid.

\section{CONCLUDING REMARKS}

Interacting cosmology has attracted the interest of the literature, since on one hand it cannot be excluded from the field theoretical point of view, and on the other hand it may offer a solution to the coincidence problem. However, almost all phenomenologically introduced interacting functions have constant sign, namely the energy flow maintains its direction throughout the whole universe evolution.

In the present work we investigated sign-changeable interacting scenarios, in which the interaction function, and thus the energy flow, changes sign during the evolution of the universe, since there is not any theoretical reason of not considering such forms. We considered various models and we extracted the involved equations at both the background and perturbation levels. Then we used various data combinations from cosmic microwave background (CMB), baryon acoustic oscillations (BAO), Supernovae Type Ia (SNIa) and cosmic chronometers (CC) in order to constrain the model parameters. Finally, we performed a Bayesian analysis in order to compare the fitting efficiency of the examined models with the reference $\Lambda$ CDM paradigm.

For both examined sign-changed interacting models, namely $Q=3 H \xi\left(\rho_{c}-\rho_{x}\right)$ and $Q=3 H\left(\alpha \rho_{c}-\beta \rho_{x}\right)$, we found that the dark-energy equation-of-state parameter 

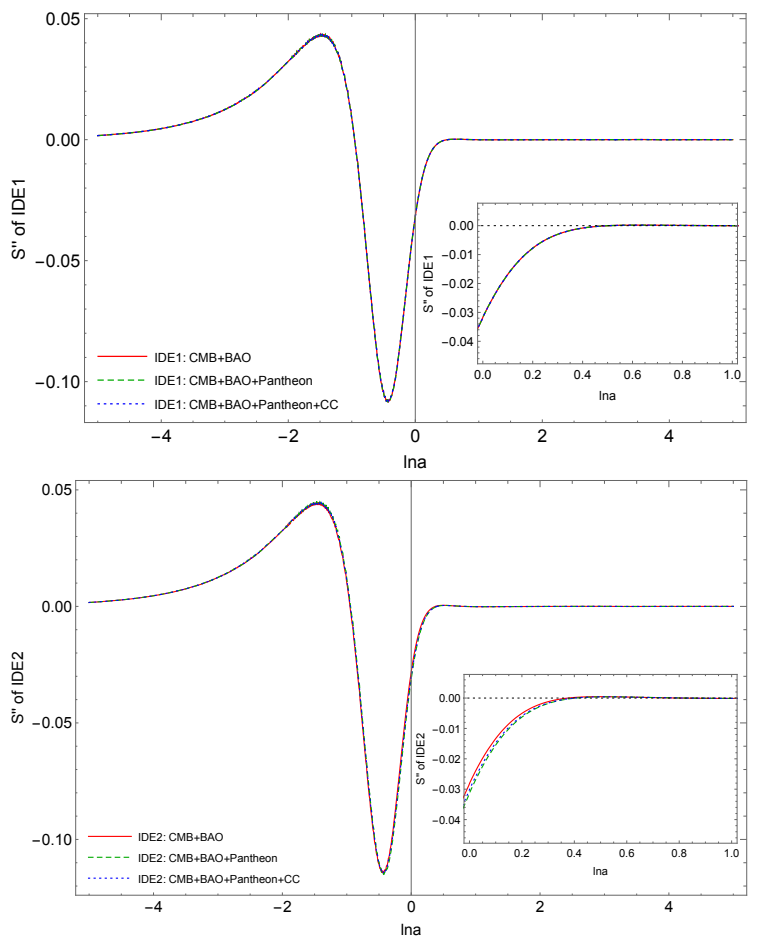

FIG. 15: The evolution of the second derivative of the total entropy $S^{\prime \prime}$ according to (35), for IDE1 (upper graph) and IDE2 (lower graph), for the combined analyses $C M B+B A O$, $C M B+B A O+$ Pantheon and $C M B+B A O+$ Pantheon $+C C$. In the subgraph we focus on late times, namely at present epoch $(\ln a=0)$ and in the future, where we can see that $S^{\prime \prime}<0$ and thus the entropy is convex as required for thermodynamic equilibrium.

$w_{x}$ prefers the phantom regime for all datasets, at more than $2 \sigma$. Concerning the coupling parameters we saw that although the best-fit values might be non-zero, the zero value, namely no interaction, is included within $1 \sigma$. Moreover, we showed that this results is maintained if we impose $w_{x}$ to take the cosmological constant value -1 , namely considering the interacting vacuum model.

We proceeded by examining the effect of the interaction on the CMB TT and matter power spectra. As we showed, while from the simple CMB TT spectra it is hard to distinguish the interacting case from $\Lambda \mathrm{CDM}$ scenario, from the residual plot (with reference to $\Lambda \mathrm{CDM}$ model) one can indeed trace a distinction between the interacting and non-interacting cosmologies, mainly in the lower multipoles. Similarly, the simple matter power spectra cannot be used to examine the interaction, however using the corresponding residual graphs we showed that the deviation from the non-interacting $\Lambda \mathrm{CDM}$ cosmology is clear. The fact that the residual spectra plots can be used to distinguish the models from $\Lambda \mathrm{CDM}$ paradigm, even if at the background level the latter is allowed withing $1 \sigma$ $\mathrm{CL}$, is one of the main results of the present work.

Concerning $H_{0}$, we saw that in all cases its obtained values are slightly higher compared to the $\Lambda \mathrm{CDM}$-based Planck estimation, and thus the $H_{0}$ tension seems to be alleviated as a result of the interaction, although only partially. Hence, we deduce that although the interaction may be small, it is adequate to alleviate the $H_{0}$ tension. Nevertheless, concerning $\sigma_{8}$ tension we found that the present sign changeable interaction models are not able to release it. These features show that sign-changeable interacting scenarios might be worthy for further investigations.

Finally, we examined the validity of the laws of thermodynamics in a universe with sign-changeable interactions in the dark sector. As we saw, the generalized second law is always satisfied, namely the total entropy, constituting from all fluids as well as from the horizon entropy, is always increasing. Additionally, its second derivative becomes negative at late times, which implies that the universe tends towards thermodynamic equilibrium.

Last but not least, it is interesting to study the sign changeable models in extended parameter spaces, allowing the spatial curvature to be non-zero. The inclusion of massive neutrinos could also be appealing. These projects are left for future investigation.

\section{Acknowledgments}

The authors would like to thank an anonymous referee for important comments that helped to improve the manuscript substantially. SP acknowledges the research funding through the Faculty Research and Professional Development Fund (FRPDF) Scheme of Presidency University, Kolkata, India. WY acknowledges the support from the National Natural Science Foundation of China under Grants No. 11705079 and No. 11647153. This article is based upon work from CANTATA COST (European Cooperation in Science and Technology) action CA15117, EU Framework Programme Horizon 2020.
[1] P. A. R. Ade et al. [Planck Collaboration], Planck 2015 results. XIII. Cosmological parameters, Astron. Astrophys. 594, A13 (2016) arXiv:1502.01589 [astroph.CO]].

[2] L. Amendola, Coupled Quintessence, Phys. Rev. D 62, 043511 (2000) arXiv:astro-ph/9908023.

[3] D. Pavón and W. Zimdahl, Holographic dark energy and cosmic coincidence, Phys. Lett. B 628, 206 (2005) arXiv:gr-qc/0505020.

[4] S. del Campo, R. Herrera and D. Pavón, Toward a solution of the coincidence problem, Phys. Rev. D 78, 021302 (2008) arXiv:0806.2116 [astro-ph]].

[5] Yu. L. Bolotin, A. Kostenko, O. A. Lemets and D.A. Yerokhin, Cosmological Evolution With Interaction Be- 
tween Dark Energy And Dark Matter, Int. J. Mod. Phys. D 24, 1530007 (2015) arXiv:1310.0085 [astro-ph.CO].

[6] B. Wang, E. Abdalla, F. Atrio-Barandela and D. Pavón, Dark Matter and Dark Energy Interactions: Theoretical Challenges, Cosmological Implications and Observational Signatures, Rept. Prog. Phys. 79 (2016) no.9, 096901 arXiv:1603.08299 [astro-ph.CO]].

[7] E. J. Copeland, M. Sami and S. Tsujikawa, Dynamics of dark energy, Int. J. Mod. Phys. D 15, 1753 (2006) hep-th/0603057.

[8] Y. F. Cai, E. N. Saridakis, M. R. Setare and J. Q. Xia, Quintom Cosmology: Theoretical implications and observations, Phys. Rept. 493, 1 (2010) arXiv:0909.2776 [hep-th]].

[9] S. Nojiri and S. D. Odintsov, Unified cosmic history in modified gravity: from $F(R)$ theory to Lorentz non-invariant models, Phys. Rept. 505, 59 (2011) arXiv:1011.0544 [gr-qc]].

[10] S. Capozziello and M. De Laurentis, Extended Theories of Gravity, Phys. Rept. 509, 167 (2011) arXiv:1108.6266 [gr-qc]].

[11] S. Nojiri, S. D. Odintsov and V. K. Oikonomou, Modified Gravity Theories on a Nutshell: Inflation, Bounce and Late-time Evolution, Phys. Rept. 692, 1 (2017) arXiv:1705.11098 [gr-qc]].

[12] S. Basilakos and M. Plionis, Is the Interacting Dark Matter Scenario an Alternative to Dark Energy?, Astron. Astrophys. 507, 47 (2009) arXiv:0807.4590 [astro$\mathrm{ph}]$.

[13] S. Basilakos and M. Plionis, Interacting Dark Matter as an Alternative to Dark Energy, AIP Conf. Proc. 1241, no. 1, 721 (2010) arXiv:0911.2310 [astro-ph.CO]].

[14] V. Salvatelli, N. Said, M. Bruni, A. Melchiorri and D. Wands, Indications of a late-time interaction in the dark sector, Phys. Rev. Lett. 113, no. 18, 181301 (2014) arXiv:1406.7297 [astro-ph.CO]].

[15] S. Basilakos, D. Polarski and J. Solá, Generalizing the running vacuum energy model and comparing with the entropic-force models, Phys. Rev. D 86, 043010 (2012) arXiv:1204.4806 [gr-qc]].

[16] J. Solà, A. Gómez-Valent and J. d. C. Pérez, First evidence of running cosmic vacuum: challenging the concordance model, Astrophys. J. 836, no. 1, 43 (2017) arXiv:1602.02103 [astro-ph.CO].

[17] X. D. Xu, B. Wang, P. Zhang and F. Atrio-Barandela, The effect of Dark Matter and Dark Energy interactions on the peculiar velocity field and the kinetic Sunyaev-Zel'dovich effect, JCAP 1312, 001 (2013) arXiv:1308.1475 [astro-ph.CO]].

[18] A. A. Costa, X. D. Xu, B. Wang, E. G. M. Ferreira and E. Abdalla, Testing the Interaction between Dark Energy and Dark Matter with Planck Data, Phys. Rev. D 89, no.10, 103531 (2014) arXiv:1311.7380 [astroph.COl].

[19] S. Basilakos and J. Sola, Effective equation of state for running vacuum: mirage quintessence and phantom dark energy, Mon. Not. Roy. Astron. Soc. 437, no. 4, 3331 (2014) arXiv:1307.4748 [astro-ph.CO]].

[20] E. J. Ruiz and D. Huterer, Testing the dark energy consistency with geometry and growth, Phys. Rev. D 91, 063009 (2015) arXiv:1410.5832 [astro-ph.CO]].

[21] T. Yang, Z. K. Guo and R. G. Cai, Reconstructing the interaction between dark energy and dark matter using Gaussian Processes, Phys. Rev. D 91, no. 12, 123533
(2015) arXiv:1505.04443 [astro-ph.CO]].

[22] Y. H. Li, J. F. Zhang and X. Zhang, Testing models of vacuum energy interacting with cold dark matter, Phys. Rev. D 93, no. 2, 023002 (2016) arXiv:1506.06349 [astro-ph.CO]].

[23] J. Väliviita and E. Palmgren, Distinguishing interacting dark energy from $w C D M$ with $C M B$, lensing, and baryon acoustic oscillation data, JCAP 1507, 015 (2015) arXiv:1504.02464 [astro-ph.CO]].

[24] M. Eingorn and C. Kiefer, Scalar perturbations in cosmological models with dark energy - dark matter interaction, JCAP 1507, 036 (2015) arXiv:1502.06912 [gr-qc]].

[25] S. Pan, S. Bhattacharya and S. Chakraborty, An analytic model for interacting dark energy and its observational constraints, Mon. Not. Roy. Astron. Soc. 452, 3038 (2015) arXiv:1210.0396 [gr-qc]].

[26] R. C. Nunes, S. Pan and E. N. Saridakis, New constraints on interacting dark energy from cosmic chronometers, Phys. Rev. D 94, no. 2, 023508 (2016) arXiv:1605.01712 [astro-ph.CO]].

[27] C. Caprini and N. Tamanini, Constraining early and interacting dark energy with gravitational wave standard sirens: the potential of the eLISA mission, JCAP 1610, no. 10, 006 (2016) arXiv:1607.08755 [astro-ph.CO]].

[28] W. Yang, S. Pan and D. F. Mota, Novel approach toward the large-scale stable interacting dark-energy models and their astronomical bounds, Phys. Rev. D 96, no. 12, 123508 (2017) arXiv:1709.00006 [astro-ph.CO]].

[29] W. Yang, S. Pan and J. D. Barrow, Large-scale Stability and Astronomical Constraints for Coupled DarkEnergy Models, Phys. Rev. D 97, no. 4, 043529 (2018) arXiv:1706.04953 [astro-ph.CO]].

[30] S. Kumar and R. C. Nunes, Echo of interactions in the dark sector, Phys. Rev. D 96, no. 10, 103511 (2017) arXiv:1702.02143 [astro-ph.CO]].

[31] E. Di Valentino, A. Melchiorri and O. Mena, Can interacting dark energy solve the $H_{0}$ tension?, Phys. Rev. D 96, no. 4, 043503 (2017) arXiv:1704.08342 [astroph.CO].

[32] W. Yang, S. Pan, E. Di Valentino, R. C. Nunes, S. Vagnozzi and D. F. Mota, Tale of stable interacting dark energy, observational signatures, and the $H_{0}$ tension,, JCAP 1809, 019, (2018) arXiv:1805.08252 [astro-ph.CO]].

[33] W. Yang, A. Mukherjee, E. Di Valentino and S. Pan, Interacting dark energy with time varying equation of state and the $H_{0}$ tension, Phys. Rev. D 98, no. 12, 123527 (2018) arXiv:1809.06883 [astro-ph.CO]].

[34] S. Kumar, R. C. Nunes and S. K. Yadav, Dark sector interaction: a remedy of the tensions between $C M B$ and LSS data, arXiv:1903.04865 [astro-ph.CO].

[35] C. Van De Bruck and J. Mifsud, Searching for dark matter - dark energy interactions: going beyond the conformal case, Phys. Rev. D 97, no. 2, 023506 (2018) arXiv:1709.04882 [astro-ph.CO]].

[36] A. Pourtsidou and T. Tram, Reconciling CMB and structure growth measurements with dark energy interactions, Phys. Rev. D 94, 043518 (2016) arXiv:1604.04222 [astro-ph.CO].

[37] V. Faraoni, J. B. Dent and E. N. Saridakis, Covariantizing the interaction between dark energy and dark matter, Phys. Rev. D 90, no. 6, 063510 (2014) arXiv:1405.7288 [gr-qc]].

[38] V. F. Mukhanov, H. A. Feldman and R. H. Bran- 
denberger, Theory of cosmological perturbations, Phys. Rept. 215, 203 (1992).

[39] C. P. Ma and E. Bertschinger, Cosmological perturbation theory in the synchronous and conformal Newtonian gauges, Astrophys. J. 455, 7 (1995) arXiv:astro$\mathrm{ph} / 9506072$.

[40] K. A. Malik and D. Wands, Cosmological perturbations, Phys. Rept. 475, 1 (2009) arXiv:0809.4944 [astro-ph]].

[41] J. Väliviita, E. Majerotto and R. Maartens, Instability in interacting dark energy and dark matter fluids, JCAP 0807, 020 (2008) arXiv:0804.0232 [astro-ph]].

[42] E. Majerotto, J. Valiviita and R. Maartens, Adiabatic initial conditions for perturbations in interacting dark energy models, Mon. Not. Roy. Astron. Soc. 402, 2344 (2010) arXiv:0907.4981 [astro-ph.CO]].

[43] T. Clemson, K. Koyama, G. B. Zhao, R. Maartens and J. Valiviita, Interacting Dark Energy - constraints and degeneracies, Phys. Rev. D 85, 043007 (2012) arXiv:1109.6234 [astro-ph.CO]].

[44] Y. Wang, D. Wands, G. B. Zhao and L. Xu, PostPlanck constraints on interacting vacuum energy, Phys. Rev. D 90, no. 2, 023502 (2014) arXiv:1404.5706] [astroph.CO]].

[45] J. Ryan, Y. Chen and B. Ratra, Baryon acoustic oscillation, Hubble parameter, and angular size measurement constraints on the Hubble constant, dark energy $d y$ namics, and spatial curvature, arXiv:1902.03196 [astroph.CO].

[46] S. Mitra, C. G. Park, T. R. Choudhury and B. Ratra, First study of reionization in tilted flat and untilted non-flat dynamical dark energy inflation models, Mon. Not. Roy. Astron. Soc. 487, no. 4, 5118 (2019) arXiv:1901.09927 [astro-ph.CO]].

[47] C. G. Park and B. Ratra, Measuring the Hubble constant and spatial curvature from supernova apparent magnitude, baryon acoustic oscillation, and Hubble parameter data, arXiv:1809.03598 [astro-ph.CO].

[48] C. G. Park and B. Ratra, Observational constraints on the tilted spatially-flat and the untilted nonflat $\phi C D M$ dynamical dark energy inflation models, Astrophys. J. 868, no. 2, 83 (2018) arXiv:1807.07421 [astro-ph.CO]].

[49] J. Ryan, S. Doshi and B. Ratra, Constraints on dark energy dynamics and spatial curvature from Hubble parameter and baryon acoustic oscillation data, Mon. Not. Roy. Astron. Soc. 480, no. 1, 759 (2018) arXiv:1805.06408 [astro-ph.CO]].

[50] C. G. Park and B. Ratra, Observational constraints on the tilted flat-XCDM and the untilted nonflat XCDM $d y$ namical dark energy inflation parameterizations, Astrophys. Space Sci. 364, no. 5, 82 (2019) arXiv:1803.05522 [astro-ph.CO]].

[51] H. Yu, B. Ratra and F. Y. Wang, Hubble Parameter and Baryon Acoustic Oscillation Measurement Constraints on the Hubble Constant, the Deviation from the Spatially Flat $\Lambda C D M$ Model, the DecelerationAcceleration Transition Redshift, and Spatial Curvature, Astrophys. J. 856, no. 1, 3 (2018) arXiv:1711.03437 [astro-ph.CO]].

[52] O. Farooq, F. R. Madiyar, S. Crandall and B. Ratra, Hubble Parameter Measurement Constraints on the Redshift of the Decelerationacceleration Transition, Dynamical Dark Energy, and Space Curvature, Astrophys. J. 835, no. 1, 26 (2017) arXiv:1607.03537 [astro-ph.CO]].

[53] Y. Chen, B. Ratra, M. Biesiada, S. Li and Z. H. Zhu, Constraints on non-flat cosmologies with massive neu- trinos after Planck 2015, Astrophys. J. 829, no. 2, 61 (2016) arXiv:1603.07115 [astro-ph.CO]].

[54] A. P. Billyard and A. A. Coley, Interactions in scalar field cosmology, Phys. Rev. D 61, 083503 (2000) arXiv:astro-ph/9908224.

[55] J. D. Barrow and T. Clifton, Cosmologies with energy exchange, Phys. Rev. D 73, 103520 (2006) grqc/0604063.

[56] W. Zimdahl and D. Pavón, Interacting holographic dark energy, Class. Quant. Grav. 24, 5461 (2007) astro$\mathrm{ph} / 0606555$.

[57] E. N. Saridakis, Holographic Dark Energy in Braneworld Models with a Gauss-Bonnet Term in the Bulk. Interactin $g$ Behavior and the $w=-1$ Crossing, Phys. Lett. B 661, 335 (2008) arXiv:0712.3806 [gr-qc]].

[58] J. H. He and B. Wang, Effects of the interaction between dark energy and dark matter on cosmological parameters, JCAP 0806, 010 (2008) arXiv:0801.4233 [astro$\mathrm{ph}]$.

[59] M. Quartin, M. O. Calvao, S. E. Joras, R. R. R. Reis and I. Waga, Dark Interactions and Cosmological FineTuning, JCAP 0805, 007 (2008) arXiv:0802.0546] [astroph].

[60] X. m. Chen, Y. g. Gong and E. N. Saridakis, Phasespace analysis of interacting phantom cosmology, JCAP 0904, 001 (2009) arXiv:0812.1117 [gr-qc]].

[61] L. P. Chimento, Linear and nonlinear interactions in the dark sector, Phys. Rev. D 81, 043525 (2010) arXiv:0911.5687 [astro-ph.CO].

[62] M. Jamil, E. N. Saridakis and M. R. Setare, Thermodynamics of dark energy interacting with dark matter and radiation, Phys. Rev. D 81, 023007 (2010) arXiv:0910.0822 [hep-th]].

[63] J. Väliviita, R. Maartens and E. Majerotto, Observational constraints on an interacting dark energy model, Mon. Not. Roy. Astron. Soc. 402, 2355 (2010) arXiv:0907.4987 [astro-ph.CO].

[64] M. B. Gavela, L. Lopez Honorez, O. Mena and S. Rigolin, Dark Coupling and Gauge Invariance, JCAP 1011, 044 (2010) arXiv:1005.0295 [astro-ph.CO]].

[65] M. Baldi, Early massive clusters and the bouncing coupled dark energy, Mon. Not. Roy. Astron. Soc. 420, 430 (2012) arXiv:1107.5049 [astro-ph.CO]].

[66] M. Thorsrud, D. F. Mota and S. Hervik, Cosmology of a Scalar Field Coupled to Matter and an IsotropyViolating Maxwell Field, JHEP 1210, 066 (2012) arXiv:1205.6261 [hep-th]].

[67] S. Pan and S. Chakraborty, Will there be again a transition from acceleration to deceleration in course of the dark energy evolution of the universe?, Eur. Phys. J. C 73, 2575 (2013) arXiv:1303.5602 [gr-qc].

[68] W. Yang and L. Xu, Coupled dark energy with perturbed Hubble expansion rate, Phys. Rev. D 90, 083532 (2014) arXiv:1409.5533 [astro-ph.CO].

[69] W. Yang and L. Xu, Cosmological constraints on interacting dark energy with redshift-space distortion after Planck data, Phys. Rev. D 89, 083517 (2014) arXiv:1401.1286 [astro-ph.CO].

[70] R. C. Nunes and E. M. Barboza, Dark matter-dark energy interaction for a time-dependent EoS parameter, Gen. Rel. Grav. 46, 1820 (2014) arXiv:1404.1620][astroph.CO]].

[71] S. Pan and S. Chakraborty, A cosmographic analysis of holographic dark energy models, Int. J. Mod. Phys. D 
23, 1450092 (2014) arXiv:1410.8281 [gr-qc].

[72] X. m. Chen, Y. Gong, E. N. Saridakis and Y. Gong, Time-dependent interacting dark energy and transient acceleration, Int. J. Theor. Phys. 53, 469 (2014) arXiv:1111.6743 [astro-ph.CO].

[73] Y. H. Li and X. Zhang, Large-scale stable interacting dark energy model: Cosmological perturbations and observational constraints, Phys. Rev. D 89, no. 8, 083009 (2014) arXiv:1312.6328 [astro-ph.CO]].

[74] G. Kofinas, E. Papantonopoulos and E. N. Saridakis, Modified BransDicke cosmology with matter-scalar field interaction, Class. Quant. Grav. 33, no. 15, 155004 (2016) arXiv:1602.02687 [gr-qc]].

[75] A. Mukherjee and N. Banerjee, In search of the dark matter dark energy interaction: a kinematic approach, Class. Quant. Grav. 34, 035016 (2017) arXiv:1610.04419 [astro-ph.CO]].

[76] S. Pan and G. S. Sharov, A model with interaction of dark components and recent observational data, Mon. Not. Roy. Astron. Soc. 472, 4736 (2017) arXiv:1609.02287 [gr-qc]].

[77] W. Yang, N. Banerjee and S. Pan, Constraining a dark matter and dark energy interaction scenario with a dynamical equation of state, Phys. Rev. D 95, no.12, 123527 (2017) arXiv:1705.09278 [astro-ph.CO]].

[78] G. S. Sharov, S. Bhattacharya, S. Pan, R. C. Nunes and S. Chakraborty, A new interacting two fluid model and its consequences, Mon. Not. Roy. Astron. Soc. 466, no. 3, 3497 (2017) arXiv:1701.00780 [gr-qc]].

[79] S. Pan, A. Mukherjee and N. Banerjee, Astronomical bounds on a cosmological model allowing a general interaction in the dark sector, Mon. Not. Roy. Astron. Soc. 477, 1189 (2018) arXiv:1710.03725 [astro-ph.CO]].

[80] W. Yang, S. Pan, L. Xu and D. F. Mota, Effects of anisotropic stress in interacting dark matter dark energy scenarios, Mon. Not. Roy. Astron. Soc. 482, no. 2, 1858 (2019) arXiv:1804.08455 [astro-ph.CO]].

[81] W. Yang, S. Pan and A. Paliathanasis, Cosmological constraints on an exponential interaction in the dark sector, Mon. Not. Roy. Astron. Soc. 482, no. 1, 1007 (2019) arXiv:1804.08558 [gr-qc]].

[82] R. von Marttens, L. Casarini, D. F. Mota and W. Zimdahl, Cosmological constraints on parametrized interacting dark energy, Phys. Dark Univ. 23, 100248 (2019) arXiv:1807.11380 [astro-ph.CO]].

[83] W. Yang, N. Banerjee, A. Paliathanasis and S. Pan, Reconstructing the dark matter and dark energy interaction scenarios from observations, arXiv:1812.06854 [astro-ph.CO].

[84] M. Asghari, J. B. Jiménez, S. Khosravi and D. F. Mota, On structure formation from a small-scales-interacting dark sector, arXiv:1902.05532 [astro-ph.CO].

[85] S. Ikeda, E. N. Saridakis, P. C. Stavrinos and A. Triantafyllopoulos, Cosmology of Lorentz fiber-bundle induced scalar-tensor theories, arXiv:1907.10950 [gr-qc].

[86] C. Y. Sun and R. H. Yue, New Interaction between Dark Energy and Dark Matter Changes Sign during Cosmological Evolution, Phys. Rev. D 85, 043010 (2012) arXiv:1009.1214 [gr-qc]].

[87] H. Wei, Cosmological Constraints on the SignChangeable Interactions, Commun. Theor. Phys. 56, 972 (2011) arXiv:1010.1074 [gr-qc]].

[88] M. Forte, On extended sign-changeable interactions in the dark sector, Gen. Rel. Grav. 46, no. 10, 1811 (2014)
arXiv:1311.3921 [gr-qc]].

[89] J. J. Guo, J. F. Zhang, Y. H. Li, D. Z. He and X. Zhang, Probing the sign-changeable interaction between dark energy and dark matter with current observations, Sci. China Phys. Mech. Astron. 61, no. 3, 030011 (2018) arXiv:1710.03068 [astro-ph.CO]].

[90] F. Arevalo, A. Cid, L. P. Chimento and P. Mella, On sign-changeable interaction in FLRW cosmology, arXiv:1901.04300 [gr-qc].

[91] R. Adam et al. [Planck Collaboration], Planck 2015 results. I. Overview of products and scientific results, Astron. Astrophys. 594, A1 (2016) arXiv:1502.01582 [astro-ph.CO]].

[92] N. Aghanim et al. [Planck Collaboration], Planck 2015 results. XI. CMB power spectra, likelihoods, and robustness of parameters, Astron. Astrophys. 594, A11 (2016) arXiv:1507.02704 [astro-ph.CO]].

[93] D. M. Scolnic et al., The Complete Light-curve Sample of Spectroscopically Confirmed SNe Ia from Pan-STARRS1 and Cosmological Constraints from the Combined Pantheon Sample, Astrophys. J. 859, no. 2, 101 (2018) doi:10.3847/1538-4357/aab9bb arXiv:1710.00845 [astro-ph.CO]].

[94] F. Beutler et al., The 6dF Galaxy Survey: Baryon Acoustic Oscillations and the Local Hubble Constant, Mon. Not. Roy. Astron. Soc. 416, 3017 (2011) arXiv:1106.3366 [astro-ph.CO]].

[95] A. J. Ross, L. Samushia, C. Howlett, W. J. Percival, A. Burden and M. Manera, The clustering of the SDSS DR7 main Galaxy sample I. A 4 per cent distance measure at $z=0.15$, Mon. Not. Roy. Astron. Soc. 449, no. 1, 835 (2015) arXiv:1409.3242 [astro-ph.CO]].

[96] H. Gil-Marín et al., The clustering of galaxies in the SDSS-III Baryon Oscillation Spectroscopic Survey: $B A O$ measurement from the $L O S$-dependent power spectrum of DR12 BOSS galaxies, Mon. Not. Roy. Astron. Soc. 460, no. 4, 4210 (2016) arXiv:1509.06373 [astroph.CO]].

[97] M. Moresco et al., A 6\% measurement of the Hubble parameter at $z \sim 0.45$ : direct evidence of the epoch of cosmic re-acceleration, JCAP 1605, 014 (2016) arXiv:1601.01701 [astro-ph.CO]].

[98] A. Lewis and S. Bridle, Cosmological parameters from $C M B$ and other data: A Monte Carlo approach, Phys. Rev. D 66, 103511 (2002) arXiv:astro-ph/0205436.

[99] A. Lewis, A. Challinor and A. Lasenby, Efficient computation of CMB anisotropies in closed FRW models, Astrophys. J. 538, 473 (2000) arXiv:astro-ph/9911177.

[100] A. Gelman and D. Rubin, Inference from iterative simulation using multiple sequences, Statistical Science 7, 457 (1992).

[101] N. Aghanim et al. [Planck Collaboration], Planck 2018 results. VI. Cosmological parameters, arXiv:1807.06209 [astro-ph.CO].

[102] H. Steigerwald, J. Bel and C. Marinoni, Probing non-standard gravity with the growth index: a background independent analysis, JCAP 1405, 042 (2014) arXiv:1403.0898 [astro-ph.CO]].

[103] R. E. Kass and A. E. Raftery, Bayes Factors, J. Am. Statist. Assoc. 90, no.430, 773 (1995).

[104] A. Heavens, Y. Fantaye, A. Mootoovaloo, H. Eggers, Z. Hosenie, S. Kroon and E. Sellentin, Marginal Likelihoods from Monte Carlo Markov Chains, arXiv:1704.03472 [stat.CO]. 
[105] A. Heavens, Y. Fantaye, E. Sellentin, H. Eggers, Z. Hosenie, S. Kroon and A. Mootoovaloo, No evidence for extensions to the standard cosmological model, Phys. Rev. Lett. 119, no. 10, 101301 (2017) arXiv:1704.03467 [astro-ph.CO]].

[106] W. Yang, S. Pan, R. Herrera and S. Chakraborty, Largescale (in) stability analysis of an exactly solved coupled dark-energy model, Phys. Rev. D 98, no. 4, 043517 (2018) arXiv:1808.01669 [gr-qc]].

[107] A. G. Riess et al., A 2.4\% Determination of the Local Value of the Hubble Constant, Astrophys. J. 826, no. 1, 56 (2016) arXiv:1604.01424 [astro-ph.CO]].

[108] G. W. Gibbons and S. W. Hawking, Cosmological Event Horizons, Thermodynamics, and Particle Creation, Phys. Rev. D 15, 2738 (1977).

[109] T. Jacobson, Thermodynamics of space-time: The Einstein equation of state, Phys. Rev. Lett. 75, 1260 (1995) gr-qc/9504004.

[110] T. Padmanabhan, Cosmological constant: The Weight of the vacuum, Phys. Rept. 380, 235 (2003) hepth/0212290.

[111] D. Bak and S. J. Rey, Cosmic holography, Class. Quant. Grav. 17, L83 (2000) hep-th/9902173.

[112] R. G. Cai and S. P. Kim, First law of thermodynamics and Friedmann equations of FriedmannRobertson-Walker universe, JHEP 0502, 050 (2005) hep-th/0501055.

[113] J. D. Bekenstein, Generalized second law of thermodynamics in black hole physics, Phys. Rev. D 9, 3292 (1974).

[114] A. V. Frolov and L. Kofman, Inflation and de Sit- ter thermodynamics, JCAP 0305, 009 (2003) hepth/0212327.

[115] U. H. Danielsson, Transplanckian energy production and slow roll inflation, Phys. Rev. D 71, 023516 (2005) hepth/0411172.

[116] R. Bousso, Cosmology and the S-matrix, Phys. Rev. D 71, 064024 (2005) hep-th/0412197.

[117] M. Akbar and R. G. Cai, Thermodynamic Behavior of Friedmann Equations at Apparent Horizon of FRW Universe, Phys. Rev. D 75, 084003 (2007) hepth/0609128.

[118] M. Jamil, E. N. Saridakis and M. R. Setare, The generalized second law of thermodynamics in Horava-Lifshitz cosmology, JCAP 1011, 032 (2010) arXiv:1003.0876 [hep-th]].

[119] J. P. Mimoso and D. Pavn, Considerations on the thermal equilibrium between matter and the cosmic horizon, Phys. Rev. D 94, no. 10, 103507 (2016) arXiv:1610.07788 [gr-qc]].

[120] N. Radicella and D. Pavón, A thermodynamic motivation for dark energy, Gen. Rel. Grav. 44, 685 (2012) arXiv:1012.0474 [gr-qc]].

[121] S. del Campo, I. Duran, R. Herrera and D. Pavón, Three thermodynamically-based parameterizations of the deceleration parameter, Phys. Rev. D 86, 083509 (2012) arXiv:1209.3415 [gr-qc]].

[122] M. Gonzalez-Espinoza and D. Pavón, Does the second law hold at cosmic scales?, Mon. Not. Roy. Astron. Soc. 484, no. 3, 2924 (2019) arXiv:1902.06651 [astroph.CO]]. 